\title{
A new model for rate-independent hysteresis in permanent magnets
}

\author{
Giorgio Gentili ${ }^{a}$, Claudio Giorgi ${ }^{b}{ }^{*}$ \\ ${ }^{a}$ Dipartimento di Matematica, Università di Bologna, 40127 Bologna, Italy \\ b Dipartimento di Matematica, Università di Brescia, Via Valotti 9, 25133 Brescia, Italy
}

Received 13 January 2000; accepted 21 April 2000

(Communicated by E.S. ŞUHUBI)

\begin{abstract}
A one-dimensional memory-based model of magnetic materials exhibiting hysteresis loops is proposed here. Mainly, we suggest a modification of the Duhem model investigated by Coleman and Hodgdon in connection with isoperms in order to better fit their real behaviour. Many properties fulfilled by the original model are preserved here. For instance, the presence of the major loop, bounding all hysteresis paths, the existence and uniqueness of asymptotically stable periodic solutions (primitive loops), the existence of a "universal" demagnetisation process (a suitable alternating magnetic field, with slowly decreasing amplitude, reproducing the "virgin state"). On the other hand, a few mathematical features are common to the Preisach model and allow the corresponding hysteretic functional to avoid effects, like self-magnetisation, which are typical of the Duhem models but physically unsound. In particular, equilibrium states are stable with respect to noises of small amplitude (static vibro-stability). (C) 2001 Elsevier Science Ltd. All rights reserved.
\end{abstract}

Keywords: Hysteresis; Rate-independent models; Ordinary differential equations; Asymptotic cycles; Alternating demagnetisation

\section{Introduction}

The electromagnetic behaviour of the most important magnetic materials for practical purposes (iron, cobalt and nichel, and their alloys) is highly non-linear. Indeed, the magnetic induction B inside them depends on the magnetic field $\mathbf{H}$ in such a way that the pair $(\mathbf{H}, \mathbf{B})$ moves along a

\footnotetext{
${ }^{*}$ Corresponding author. Fax: +39-0303715745.

E-mail address: giorgi@ing.unibs.it (C. Giorgi).
} 
smooth curve in $\mathbb{R}^{3} \times \mathbb{R}^{3}$ which changes at every "turning point" of the "input" function $\mathbf{H}(\cdot)$. The corresponding physical phenomenon, called hysteresis, is a permanent memory effect, in the sense that any magnetisation previous to the time that the sample is set up for study will be partially, but permanently, retained in both magnitude and direction. In essence, $\mathbf{B}$ at instant $t$ cannot be simply considered as function of $\mathbf{H}$ at the same instant, but it depends on the whole previous history of $\mathbf{H}$. Thus, hysteretic phenomena have to be set into the framework of materials with memory.

In literature the most common mathematical representation of hysteretic behaviour is performed by means of differential models which are also referred to as Duhem models (e.g., [14,18]) or Madelung's prehysterons [12]. These models uses a phenomenological approach and assumes that the output can only change its character when the input changes direction. They are useful in applied electromagnetism because all functions and parameters involved can be properly chosen to match experimental results. In particular, these models fit well for magnetic materials exhibiting narrow hysteresis loops, such as isoperms. In this connection, Coleman and Hodgdon in [3,4] and Hodgdon in $[9,10]$ proposed a class of differential unidimensional models accounting for most of the properties of isoperms. Unfortunately, due to their short-range memory, not all the properties of the Duhem hysteresis operator are satisfactory. Indeed, some effects provided by most of these models, like self-magnetisation, are entirely without physical foundation.

In recent years, a systematic mathematical analysis about classes of models for materials that give rise to hysteresis has been carried out by several authors. Krasnosel'skii and co-workers (e.g., [12]) devote a major effort to extending the Duhem and Ishlinskii models from piecewise monotone inputs to continuous inputs, in the framework of more general researches on nonlinear dynamical systems. Visintin [19], Brokate [2] and Mayergoyz [15] studied the properties of the classical Preisach model and proposed further refinements. Damlamian and Visintin [5], Mayergoyz [15] and Krasnosel'skii and Pokrovskii [12] extended hysteresis models to vectorvalued input-output fields. Many efforts have been especially devoted to establish existence and uniqueness of solutions for partial and functional differential equations related to hysteretic evolutive phenomena $[8,13,20,21]$. Most of these results rest heavily on a property, called vibrostability by Krasnosel'skii [11], which ensures that the hysteretic functional can be extended continuously to the normed space of continuous functions [12, Part 3]. In essence, this means the dynamical stability of input-output processes with respect to the $C^{0}$-norm. In particular, all equilibrium states (static solutions) are stable in the same norm. This is a fundamental property of permanent magnets, where any given magnetised state at equilibrium is stable under perturbations of small amplitude (noises). In other words, no spontaneous (de)magnetisation occurs. On the contrary, any magnetic model exhibiting self-magnetisation phenomena is not vibrocorrect.

Our purpose is to present here a simple memory-based approach to the modelisation of uniaxial magnetic hysteresis by mixing some features which are peculiar of the Duhem or Preisach-type model. To this end we construct a peculiar differential-hereditary model by modifying that proposed in $[3,4]$, and scrutinise its relevant properties. All properties of Coleman-Hodgdon's model are recovered (existence of the major loop, the limiting loop and the universal demagnetisation process independent of the initial state, etc.), and a partial agreement with Krasnosel'skii's notion of vibro-stability is achieved. Indeed, we prove the stability in the $C^{0}$-norm of any stationary magnetic state, so that a statically vibro-correct hysteretic functional can be constructed. In view 
of this feature that avoids self-magnetisation, we expect to better fit our model to the phenomenology of permanent magnets.

\section{Rate-independent models for hysteresis}

For the sake of simplicity, henceforth we shall restrict our attention to uniaxial fields (i.e., unidimensional phenomena), so that the magnetic field $H$ and the magnetic induction $B$ will be treated as scalar fields. We develop in the following some basic arguments concerning the mathematical modelling of hysteresis.

One-dimensional hysteresis generally leads to a non-monotone path representation, so that at least two fixed families of curves exist in the $(H, B)$-plane, one family for increasing $H$, one family for decreasing $H$. Thus, any constitutive functional for hysteresis cannot be assumed monotone. Nevertheless, it is usually assumed that when $H$ increases (decreases), then $B$ increases (decreases) so that the pair $(H, B)$ moves along the graph of a monotone curve in the $(H, B)$-plane, but this curve is changed at every turning point of $H$. If this is the case, the weaker property named piecewise monotonicity holds.

In a memory-based approach the constitutive equation for the magnetic material is a functional relation between $B(t)$ and the history of $H$. Causality forces this relation to be represented by means of a non-linear Volterra (i.e., causal) functional. Accordingly, the current magnetisation value $B(\tau), \tau \in\left[t_{0}, t\right]$, can be uniquely expressed as a functional depending on the history of $H$ up to $\tau$. This is the same approach followed in viscoelasticity and many other memory-type theories.

Magnetic materials with hysteresis, however, exhibit permanent memory, which means that not only the magnetic history of $H$ but also the initial magnetisation $B_{0}$ affects the current magnetisation value. Indeed, the initial magnetisation value is permanently retained even after a constant magnetic process of arbitrary duration. This is very far from the behaviour peculiar of viscoelasticity: there, due to the relaxation property, memory fades in time and the virgin state can always be attained, at least asymptotically, by means of a suitable path called static continuation. Therefore, the relaxation property, as assumed for viscoelastic materials, does not hold when hysteretic phenomena are involved.

The history of the magnetic field up to time $t$ is given by the pair $\left(H(t), H^{t}\right)$, where $H^{t}(s)=H(t-s), s>0$, is often called reduced history. Given any fixed initial magnetisation $B_{0}$, the constitutive relation is then expressed by

$$
B(\tau)=\mathscr{B}\left(H(\tau), H^{\tau}, B_{0}\right), \quad \tau \in\left[t_{0}, t\right]
$$

where the initial magnetisation $B_{0}$ can be interpreted as the value taken by $B$ at $\tau=-\infty$. However, due to the permanent character of the hysteretic memory, this choice is not unique. Indeed, we could assume that $B_{0}=B\left(t_{0}\right)$, as well.

Unfortunately, some undesirable, unphysical consequences arise from the permanent memory property, if unrestricted. For instance, it might be impossible to restore the situation corresponding to the initial zero-history and zero-magnetisation (henceforth, referred to as the virgin state). On the contrary, it is well known that in permanent magnets an alternating magnetic field with "slowly" decreasing amplitude, called demagnetisation, is usually applied for practical pur- 
poses in order to restore asymptotically the virgin state. To avoid this difficulty, we are forced to assume that in permanent magnets memory fades, too; but as the total variation $\lambda^{t}(s)$, not the elapsed time $s$, increases. This property will be formalised later as a generalisation of a pioneer paper by Bouc [1].

During the electromagnetic solicitation of the sample, other memory effects than hysteresis can also occur at both the microscopic and macroscopic scales, for instance relaxation and ageing. Henceforth, we assume that ferromagnetic hysteresis represents the main aspect of magnetic behaviour of the sample and we neglect any other memory phenomenon, consequently. In order to discriminate hysteresis from different memory-based models, we require that constitutive relation (2.1) fulfills the properties of rate-independence: the velocity at which the input $H(t)$ moves on the $H$-axis is only reflected in the velocity of the output $B(t)$ on the $B$-axis, but the qualitative nature of the response does not change.

This concept was widely studied by Owen and Williams in [16]; they stated that a memory functional $\mathscr{B}$ is rate-independent if it is invariant for application of any increasing homeomorphism $\varphi$ to the time variable. When this is the case, $B(t)$ depends just on the range of values assumed by $H^{t}(s), s \in \mathbb{R}^{+}$, and on the order in which these values are taken. As firstly pointed out by Pipkin and Rivlin [17] for stress-strain memory functionals, an intrinsic representation of the history can be given.

To this end, we introduce the space $B V_{\text {loc }}\left(\mathbb{R}^{+}\right)$of measurable functions with locally bounded variation, namely,

$$
B V_{\mathrm{loc}}\left(\mathbb{R}^{+}\right)=\left\{u: \mathbb{R}^{+} \rightarrow \mathbb{R}: u \in L_{\mathrm{loc}}^{1}\left(\mathbb{R}^{+}\right) \text {and } \underset{[0, T]}{\operatorname{Var}}(u)<\infty \forall T>0\right\}
$$

where

$$
\underset{[0, T]}{\operatorname{Var}}(u)=\sup _{0, T}\left\{\sum_{k=1}^{N}\left|u\left(t_{k}\right)-u\left(t_{k-1}\right)\right|: 0<t_{0}<\cdots<t_{N}<T\right\} .
$$

Following Visintin [18], we can choose as representatives of $B V_{\text {loc }}$ the functions which are left continuous in $(0, \infty)$. Since for any of these functions the limits at 0 and $T, T>0$, exist and are finite, we set

$$
L B V=\left\{u \in B V_{\text {loc }}: u \text { is left continuous in }(0, \infty)\right\}
$$

Moreover, for any $u \in L B V$ and $T>0$ we define the Lebesgue-Stieltjes measure, $\mathrm{d} u$, and the corresponding Lebesgue-Stieltjes integral operator on $[0, T], L_{\mathrm{d} u}$, as

$$
L_{\mathrm{d} u}(\varphi)=\int_{(0, T]} \varphi \mathrm{d} u \quad \forall \varphi \in C^{0}((0, T])
$$

In particular, we have 


$$
\underset{[0, T]}{\operatorname{Var}}(u)=\int_{(0, T]}|\mathrm{d} u|=\left\|L_{\mathrm{d} u}\right\|_{C^{0}((0, T])^{\prime}}
$$

By paralleling the approach of Pipkin and Rivlin [17], we claim that the magnetic-field history $H^{t}$ can be described by specifying the corresponding magnetic-field path (i.e., its intrinsic representation $\hat{H}^{t}$ ) and the rate-of-traversal of the path (i.e., the time dependence of its arc length $\lambda^{t}$ ). The latter is defined by

$$
\lambda^{t}(s)=\int_{(0, s]}\left|\mathrm{d} H^{t}\right|=\underset{[0, s]}{\operatorname{Var}}\left(H_{s}\right) .
$$

In other words, $\lambda^{t}(s), s \in \mathbb{R}^{+}$, is the total variation of $H_{s}$, the $s$-segment of $H^{t}$, since the magneticfield history is just required to be of locally bounded variation $\left(H^{t} \in L B V\right)$. Let $\Lambda^{t}$ denote the total variation of the magnetic history $H^{t}$, namely,

$$
\Lambda^{t}=\lim _{s \rightarrow+\infty} \lambda^{t}(s)=\int_{(0, \infty)}\left|\mathrm{d} H^{t}\right| .
$$

Then (2.2) allows histories to be of unbounded total variation $\left(\Lambda^{t}=+\infty\right)$, including periodic or generally alternating functions. As a consequence, the magnetic-field path $\hat{H}^{t}$ is an intrinsically continuous, piecewise monotone function of $\lambda$, implicitly given by $\hat{H}^{t}\left(\lambda^{t}(s)\right)=H^{t}(s), s \in(0, \infty)$. In particular, the pair $\left(H^{t}, H(t)\right)$ is equivalent to $\left(\hat{H}^{t}, \lambda^{t}, H(t)\right)$.

Because of the monotonicity of $\lambda^{t}$, when $H^{t}$ is continuous the relation between $s$ and $\lambda$ may be inverted so leading to

$$
\hat{H}^{t}(\lambda)=H^{t}\left(\left[\lambda^{t}\right]^{-1}(\lambda)\right), \quad \lambda \in\left[0, \Lambda^{t}\right]
$$

Thus, the graph of the magnetic-field path corresponding to a continuous history is composed by straight lines with slope +1 or -1 . On the contrary, if $H^{t}$ is discontinuous at $s_{0}$, letting $\Delta H_{0}$ the absolute value of its jump and $\lambda_{0}=\lambda^{t}\left(s_{0}\right)$ its total variation up to $s_{0}$, then $\hat{H}^{t}$ is not defined on the interval $\left(\lambda_{0}, \lambda_{0}+\Delta H_{0}\right)$. Nevertheless, we assume to complete its graph by means of straight lines (whose slope is +1 or -1 , obviously) on all intervals where the magnetic-field path is not defined because of its discontinuity. In this way $\hat{H}^{t}$ turns out to be absolutely continuous and differentiable almost everywhere with respect to $\lambda$ for all $H^{t} \in L B V$.

We summarise previous arguments by stating that rate-independence of the memory functional is equivalent to assume that $\mathscr{B}$ depends on $H^{t}$ through its magnetic-field path, $\hat{H}^{t}$, but is independent of its rate of traversal, namely,

$$
B(t)=\hat{\mathscr{B}}\left(H(t), \hat{H}^{t}, B_{0}\right) .
$$

A special formulation of this model is obtained by substituting the intrinsic representation $\hat{H}^{t}$ by the string

$$
\mathscr{H}^{t}=\left(\bar{H}_{1}, \bar{H}_{2}, \ldots, \bar{H}_{n}, \ldots\right)
$$


of the values attained by $\hat{H}^{t}$ at the end of each straight line with slope +1 or -1 (local maxima and minima for $\hat{H}^{t}$ and $H^{t}$ ). Each $\bar{H}_{n}, n \geqslant 1$, is called inversion point in that it represents the value of the field $H$ at some instant $\bar{t}_{n} \in(-\infty, t)$, called inversion time, such that, $H$ increases (decreases) before $\bar{t}_{n}$, and decreases (increases) after $\bar{t}_{n}$, either continuously or with a jump.

As shown in Fig. 1, the string $\mathscr{H}^{t}$ is completely equivalent to $\hat{H}^{t}$, so that (2.4) can be rewritten in the form

$$
\left.B(t)=\overline{\mathscr{B}}\left(H(t), \mathscr{H}^{t}, B_{0}\right)\right) .
$$

Given $t_{0}<t$, each history $H^{t}$ may be uniquely decomposed into the pair

$$
H^{t}=\left(H_{t-t_{0}}, H^{t_{0}}\right)
$$

of a reduced history $H^{t_{0}}$ and a process $H_{t-t_{0}}$ of duration $t-t_{0}$ such that

$$
H_{t-t_{0}}(s)=H\left(t_{0}+s\right)=H^{t}\left(t-t_{0}-s\right) \quad \forall s \in\left[0, t-t_{0}\right) .
$$

As a consequence, if $\hat{H}^{t_{0}}$ denotes the magnetic-field path up to $t_{0}$, and $\hat{H}_{\left[t_{0}, t\right)}$ denotes path corresponding to $H_{t-t_{0}}$, by virtue of (2.3) and (2.4) it follows:

$$
B(t)=\hat{\mathscr{B}}\left(H(t), \hat{H}^{t}, B_{0}\right)=\hat{\mathscr{B}}\left(H(t), \hat{H}_{\left[t_{0}, t\right)}, \hat{H}^{t_{0}}, B_{0}\right) .
$$

In all rate-independent models with permanent memory the magnetisation $B$ is unaffected by constant magnetic histories. That is,

$$
\hat{\mathscr{B}}\left(H_{0}, \hat{H}_{\left[t_{0}, t\right)}^{0}, \hat{H}^{t_{0}}, B_{0}\right)=B_{0} \quad \forall H_{0},
$$

where $\hat{H}_{\left[t_{0}, t\right)}^{0}$ is the constant magnetic-field path with value $H_{0}$ on $\left[t_{0}, t\right)$. Accordingly, when $H^{t}$ is held constant and equal to some value $H_{0}$ from $s=t-t_{0}$ onward, the magnetic state of the material remains unchanged up to $t_{0}$. Therefore, $B(t)=B_{0}, t \leqslant t_{0}$, and for any $t>t_{0}$ from (2.6) we have

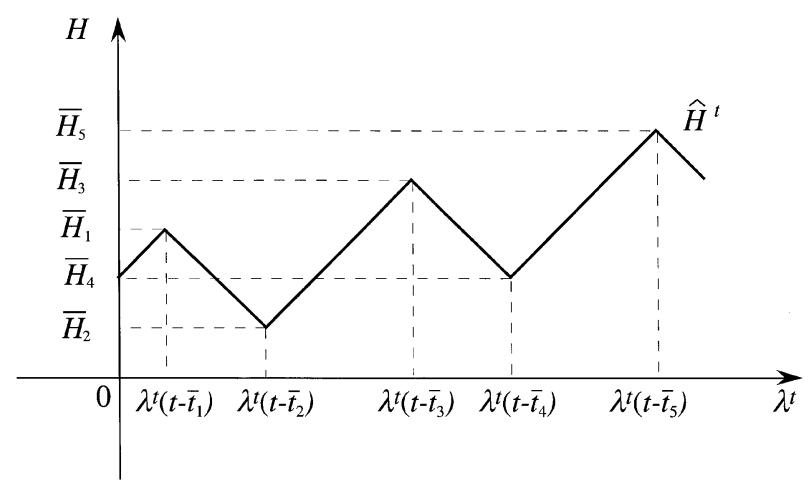

Fig. 1. The magnetic-field path $\hat{H}^{t}$ and the inversion point $\bar{H}_{k}, k \in \mathbb{N}$. 


$$
B(t)=\hat{\mathscr{B}}\left(H(t), \hat{H}_{\left[t_{0}, t\right)}, \hat{H}_{0}^{t_{0}}, B_{0}\right)
$$

where $H_{0}^{t_{0}}(s)=H_{0} \forall s>0$. Seemingly, the constant history $\left(H_{0}, H_{0}^{t_{0}}\right)$ can be summarised by the final value of $B$, namely, $B_{0}=B\left(t_{0}\right)$. Special models, however, exhibit this property for any past history, not only constant. In this case we write

$$
B(t)=\hat{\mathscr{B}}\left(H(t), \hat{H}_{\left[t_{0}, t\right)}, \hat{H}_{0}^{t_{0}}, B_{0}\right)=\hat{\mathscr{B}}\left(H(t), \hat{H}_{\left[t_{0}, t\right)}, B_{0}\right) .
$$

As we shall see in the following, this is the case if differential models are considered.

\section{Hysteresis models of the differential type}

The memory functional $\hat{\mathscr{B}}$ of a differential model is usually given to begin with only on rather narrow classes of functions. Most often it is represented by the solution of a differential equation or inclusion. Models of such a type are also referred to as Madelung's prehysterons or Duhem models. Dealing with piecewise smooth and monotone, scalar-valued histories and processes, let us define the magnetic rate process $P$ as

$$
P(t)=\dot{H}(t), \quad t \in\left[0, d_{p}\right),
$$

and assume it to be piecewise continuous. Given the initial magnetic field $H_{0}$ at time $t=0$, the magnetic process $H=\tilde{H}\left(H_{0}, P\right)$ corresponding to $H_{0}$ and $P$ is trivially defined on $\left[0, d_{p}\right)$ by

$$
H(t)=\tilde{H}\left(H_{0}, P\right)(t) \stackrel{\text { def }}{=} H_{0}+\int_{0}^{t} P(\tau) \mathrm{d} \tau .
$$

Thus, we have

$$
\underset{\left[0, d_{p}\right]}{\operatorname{Var}}(H)=\int_{\left[0, d_{p}\right)}|\mathrm{d} H|=\int_{0}^{d_{p}}|P(\tau)| \mathrm{d} \tau .
$$

The memory functional $\hat{\mathscr{B}}$ is associated to the solution $B(t)=\tilde{\mathscr{B}}\left(H_{0}, B_{0}, P_{[0, t)}\right)$ of the ordinary differential Cauchy-problem

$$
\left\{\begin{array}{l}
\dot{B}(t)=\mathscr{G}\left(\tilde{H}\left(H_{0}, P\right)(t), B(t), P(t)\right), \quad t \in\left[0, d_{p}\right], \\
B(0)=B_{0},
\end{array}\right.
$$

namely,

$$
\hat{\mathscr{B}}\left(H(t), \hat{H}_{[0, t)}, B_{0}\right)=\tilde{\mathscr{B}}\left(H_{0}, B_{0}, P_{[0, t)}\right) .
$$

As a consequence, in differential models the initial magnetic state $m_{0}$ is represented by the pair $\left(H_{0}, B_{0}\right)$. A magnetic system with memory is referred to as short-memory magnetic system [12, Part 1], 
if its state at any fixed time $t_{0}$ can be uniquely determined by the values $H_{0}=H\left(t_{0}\right), B_{0}=B\left(t_{0}\right)$ of the input and output.

The evolution of $m_{0}$ corresponding to some piecewise continuous control process $P$ is governed by the differential system

$$
\left\{\begin{array}{l}
\dot{B}(t)=\mathscr{G}(H(t), B(t), P(t)), \quad t \in\left[0, d_{p}\right] \\
\dot{H}(t)=P(t) \\
B(0)=B_{0} \\
H(0)=H_{0}
\end{array}\right.
$$

whose solution $\left(\tilde{H}\left(H_{0}, P\right), \tilde{B}\left(H_{0}, B_{0}, P\right)\right)$ defines the so-called magnetic-state transition function, $\mathscr{T}=(\tilde{H}, \tilde{B})$. It is natural to assume that the set of all feasible magnetic states is a closed, simply connected subset $\mathscr{M}$ of the $(H, B)$-plane, whose boundary $\partial \mathscr{M}$ represents the so-called major hysteresis loop. Therefore, the state transition function $\mathscr{T}$ is required to map $\mathscr{M}$ into $\mathscr{M}$.

Rate-independence property is fulfilled by (3.2) if and only if $\mathscr{G}$ is invariant under time-scale changes, i.e., $\mathscr{G}(H, B, \gamma P)=\gamma \mathscr{G}(H, B, P), \gamma \geqslant 0$. In other words, $\mathscr{G}$ must be positively homogeneous of degree 1 with respect to $P$. As a consequence, there exist two state functions, $\mathscr{G}_{1}$ and $\mathscr{G}_{2}$ such that

$$
\mathscr{G}(H, B, P)=\mathscr{G}_{1}(H, B) P+\mathscr{G}_{2}(H, B)|P|
$$

or, that is the same,

$$
\mathscr{G}(H, B, P)=\mathscr{G}_{+}(H, B) P^{+}+\mathscr{G}_{-}(H, B) P^{-},
$$

where

$$
\mathscr{G}_{+}=\mathscr{G}_{1}+\mathscr{G}_{2}, \quad \mathscr{G}_{-}=\mathscr{G}_{1}-\mathscr{G}_{2}
$$

and

$$
P^{+}=\frac{1}{2}(P+|P|), \quad P^{-}=\frac{1}{2}(P-|P|) .
$$

Introducing the so-called differential permeability,

$$
\mu=\mathscr{G}_{1}+\mathscr{G}_{2} \operatorname{sgn}(P), \quad \operatorname{sgn}(P)= \begin{cases}+1 & \text { for } P>0 \\ 0 & \text { for } P=0 \\ -1 & \text { for } P<0\end{cases}
$$

another equivalent form of (3.3) is given by

$$
\mathscr{G}(H, B, P)=\mu(H, B ; \operatorname{sgn}(P)) P .
$$

As a consequence, we have $\mu=\mathscr{G}_{+}$if $\operatorname{sgn}(P)=1, \mu=\mathscr{G}_{-}$if $\operatorname{sgn}(P)=-1$ and $\mu=\mathscr{G}_{1}$ if $P=0$. 
Piecewise monotonicity is fulfilled if both $\mathscr{G}_{+}$and $\mathscr{G}_{-}$in (3.3) are positive for any value of their arguments. In addition, assuming that they are continuous with respect to their arguments and locally Lipschitz-continuous with respect to $B$ for any $P \in L^{1}(0, T)$ there exists a unique solution $\tilde{\mathscr{B}}\left(H_{0}, B_{0}, P\right) \in W^{1,1}(0, T)$ of the Cauchy problem (3.2). As stressed in [22], for every $B_{0}$ the functional

$$
\mathscr{F}_{B_{0}}\left(H_{[0, t]}\right) \stackrel{\text { def }}{=} \hat{\mathscr{B}}\left(H(t), H_{[0, t)}, B_{0}\right)
$$

is continuous with respect to the strong topology of $W^{1, p}(0, T)$ for all $p \in[1,+\infty)$ and can be extended into a continuous operator $\tilde{\mathscr{F}}_{B_{0}}$ from $B V(0, T) \cap C^{0}([0, T])$ into itself. Such an extension is unique and some authors call them Madelung's hysteretic functional.

Unfortunately, as stressed by Krasnosel'skii (see of [12, Part 6; 18]), all differential (or Duhem) models with non-vanishing $\mathscr{G}_{2}$ term are weakly vibro-correct, but not vibro-correct at all. Apart from mathematical difficulties arising from this lack (see, for instance, [18]), it involves selfmagnetisation phenomena. In most applications to soft magnetic materials, however, the selfmagnetisation, if occurs, is negligible, in that such materials exhibit very thin hysteresis loops. On the contrary, in the mathematical modelling of permanent magnets with large loops such a shortcoming is unacceptable.

It is unquestionable that differential models are endowed with a lot of useful mathematical properties. For instance, they are (completely) controllable, because of the finite dimension of the corresponding state space. This means that starting from any initial state $m_{0} \in \mathscr{M}$ the virgin state, namely, $(H, B)=(0,0)$, is achieved by means of a suitable magnetic process of finite duration $P_{0}$ which depends on $m_{0}$. It is worth noting that such a process has nothing in common with the socalled universal demagnetisation process, an alternating magnetic field with decreasing amplitude and infinite duration which asymptotically drives the system to the virgin state whatever the initial state may be.

In the sequel a simple differential model is presented as an example $[3,4]$. For the modelling of soft magnetic materials, Coleman and Hodgdon [3,4] propose a constitutive relation of the form

$$
\dot{B}(t)=\alpha|\dot{H}(t)|[f(H(t))-B(t)]+\dot{H}(t) g(H(t))
$$

This is a special case of (3.2) and takes the compact form

$$
\frac{\mathrm{d} B}{\mathrm{~d} H}=\alpha \operatorname{sgn}(\dot{H})[f(H)-B]+g(H)
$$

In [3] Coleman and Hodgdon analyse the model assuming (see Fig. 2)

$$
f(H)=\left\{\begin{array}{ll}
a\left[H+H^{*}\right]-b H^{*} & \text { for } H<-H^{*} \\
b H & \text { for }-H^{*} \leqslant H \leqslant H^{*}, \\
a\left[H-H^{*}\right]+b H^{*} & \text { for } H>H^{*},
\end{array} \quad g(H)= \begin{cases}u & \text { for }|H| \leqslant H^{*} \\
a & \text { for }|H|>H^{*}\end{cases}\right.
$$

with $H^{*}, a, b$ and $u$ positive material constants such that $b>u \geqslant a$. This inequality is necessary and sufficient for the major hysteresis loop to enclose a region with non-zero area and have the counterclockwise orientation observed in practice. 


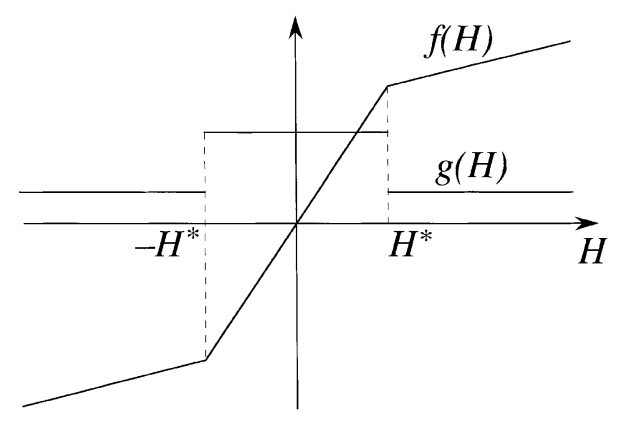

Fig. 2. Functions $f$ and $g$ of Eq. (3.7).

Integration of (3.6) and (3.7) leads to the loading curve $B_{\mathrm{L}}(H)$ and the unloading curve $B_{\mathrm{U}}(H)$ given by:

$$
\begin{aligned}
& B_{\mathrm{L}}(H)= \begin{cases}a H+H^{*}(a-b)+\mathrm{e}^{-\alpha H}\left(C_{2}-((b-u) / \alpha) \mathrm{e}^{-\alpha H^{*}}\right), & H<-H^{*}, \\
b H+\left(C_{2} \mathrm{e}^{-\alpha H}-((b-u) / \alpha)\right), & |H| \leqslant H^{*}, \\
a H-H^{*}(a-b)+\mathrm{e}^{-\alpha H}\left(C_{2}-((b-u) / \alpha) \mathrm{e}^{\alpha H^{*}}\right), & H>H^{*},\end{cases} \\
& B_{\mathrm{U}}(H)= \begin{cases}a H+H^{*}(a-b)+\mathrm{e}^{\alpha H}\left(C_{2}+((b-u) / \alpha) \mathrm{e}^{\alpha H^{*}}\right), & H<-H^{*}, \\
b H+\left(C_{2} \mathrm{e}^{\alpha H}+((b-u) / \alpha)\right), & |H| \leqslant H^{*}, \\
a H-H^{*}(a-b)+\mathrm{e}^{\alpha H}\left(C_{2}+((b-u) / \alpha) \mathrm{e}^{-\alpha H^{*}}\right), & H>H^{*}\end{cases}
\end{aligned}
$$

Observe also that the residual magnetisation $B_{\mathrm{r}}$ is given by

$$
B_{\mathrm{r}}=\frac{(b-u)}{\alpha}\left(1-\mathrm{e}^{-\alpha H^{*}}\right)
$$

and the coercive magnetic field $H_{\mathrm{c}}$ is the value that solves the equation

$$
B_{\mathrm{L}}\left(H_{\mathrm{c}},-\infty\right)=0
$$

namely,

$$
\begin{cases}b H_{\mathrm{c}}-((b-u) / \alpha)\left(1-\mathrm{e}^{-\alpha\left(H_{\mathrm{c}}+H^{*}\right)}\right)=0 & \text { if } 0<H_{\mathrm{c}} \leqslant H^{*} \\ a H_{\mathrm{c}}-H^{*}(a-b)-((b-u) / \alpha) \mathrm{e}^{-\alpha H_{\mathrm{c}}}\left(\mathrm{e}^{\alpha H^{*}}-\mathrm{e}^{-\alpha H^{*}}\right)=0 & \text { if } H_{\mathrm{c}}>H^{*}\end{cases}
$$

The corresponding energy product is given by $H_{\mathrm{c}} B_{\mathrm{r}}$.

Suitable values of the parameters $\alpha, H^{*}, a, b$ and $u$ give rise to hysteresis loops that are narrow and have the general form associated with isoperms, particularly textured isoperms and $\mathrm{Mn}-\mathrm{Zn}$ ferrites [7]. These materials are widely employed in manufacturing magnetic core because their energy loss is very small. 


\section{Hysteresis models of the functional-differential type}

We define the magnetic rate process $P$ and the magnetic history $H^{t}$ as in the previous sections. Then, given a magnetic initial history $\left(H_{0}, H^{0}\right)$, where $H_{0}=H(0)$, and a magnetic rate process $P$, we define the magnetic process $H=\tilde{H}\left(H_{0}, P\right)$ by

$$
H(t)=\tilde{H}\left(H_{0}, P_{[0, t)}\right) \stackrel{\text { def }}{=} H_{0}+\int_{0}^{t} P(\tau) \mathrm{d} \tau, \quad t \in\left[0, d_{p}\right],
$$

and the magnetic final-history $H^{d_{p}}=\hat{H}\left(H_{0}, H^{0}, P\right)$, obtained from $H^{0}$ by applying $P$, as follows:

$$
H^{d_{p}}(t)=\hat{H}\left(H_{0}, H^{0}, P_{[0, t)}\right) \stackrel{\text { def }}{=} \begin{cases}H^{0}\left(t-d_{p}\right) & \text { if } t \in\left(d_{p},+\infty\right), \\ H_{0}+\int_{0}^{d_{p}-t} P(\tau) \mathrm{d} \tau & \text { if } t \in\left(0, d_{p}\right] .\end{cases}
$$

For history-differential models the appropriate subsequent values of $B\left(d_{p}\right)$ depend not only on the magnetic final-history $\left(H^{d_{p}}, H\left(d_{p}\right)\right)$, but also on whether $H$ is increasing or decreasing after $d_{p}$. When $H$ and $B$ are smooth, scalar functions of time, this means then $\dot{B}(t)$ also depends on $\dot{H}(t)$, the time derivative of $H$ at $t$. Let

$$
B(t)=\tilde{\mathscr{B}}\left(H_{0}, H^{0}, B_{0}, P_{[0, t)}\right)
$$

be the solution of the ordinary functional-differential Cauchy problem

$$
\left\{\begin{array}{l}
\dot{B}(t)=\mathscr{G}\left(\tilde{H}\left(H_{0}, P_{[0, t)}\right), \hat{H}\left(H_{0}, H^{0}, P_{[0, t)}\right), B(t), P(t)\right), \quad t \in\left[0, d_{p}\right], \\
B(0)=B_{0} .
\end{array}\right.
$$

In this case, the memory functional $\mathscr{B}$ is represented by

$$
\mathscr{B}\left(\tilde{H}\left(H_{0}, P_{[0, t)}\right), \hat{H}\left(H_{0}, H^{0}, P_{[0, t)}\right), B_{0}\right)=\tilde{\mathscr{B}}\left(H_{0}, H^{0}, B_{0}, P_{[0, t)}\right)
$$

and the initial magnetic state is given by the triplet $m^{0}=\left(H_{0}, H^{0}, B_{0}\right)$, whose projection $m_{0}=\left(H_{0}, B_{0}\right)$ belongs to some closed and simply connected subset $\mathscr{M}$ of the $(H, B)$-plane. Let $\Sigma$ be the set of all feasible states. The evolution of $m^{0}$ in $\Sigma$ is governed by the system

$$
\left\{\begin{array}{l}
\dot{H}(t)=P(t), \quad t \in\left[0, d_{p}\right), \\
\dot{B}(t)=\mathscr{G}\left(H(t), H^{t}, B(t), P(t)\right), \quad t \in\left[0, d_{p}\right) \\
\dot{H}^{t}(s)=-(\partial / \partial s) H^{t}(s), \quad t \in\left[0, d_{p}\right), \quad s \in(0,+\infty) \\
H(0)=H_{0}, \quad B(0)=B_{0},\left.\quad H^{t}\right|_{t=0}=H^{0}
\end{array}\right.
$$

where $\left.H^{t}\right|_{t=0}$ is the backward translation by $t$ of the history $H^{t}$, namely, $\left.H^{t}\right|_{t=0}(s)=H^{t}(s+t)$, $s \in(0,+\infty)$. Solving (4.3) one obtains the related-state transition function, namely,

$$
\mathscr{T}=(\tilde{H}, \hat{H}, \bar{B}),
$$

which maps the state space $\Sigma$ into itself. 
Models like (4.3) have been proposed by Fabrizio and Giorgi in [6]. As far as we know, no specific applications of this general setting have been performed till now. In Section 5 we give a first attempt in this direction.

Remark 4.1. It is easily seen that the functional constitutive equation (4.2) is rate independent if and only if $\hat{\mathscr{G}}$ is positively homogeneous of degree 1 with respect to $P$ and depends on $H^{t}$ through its intrinsic representation $\hat{H}^{t}$, namely,

$$
\mathscr{G}\left(H(t), H^{t}, B(t), P(t)\right)=\mathscr{G}_{1}\left(H(t), \hat{H}^{t}, B(t)\right) P(t)+\mathscr{G}_{2}\left(H(t), \hat{H}^{t}, B(t)\right)|P(t)| .
$$

Using $\mathscr{H}^{t}$ as an equivalent representation for $\hat{H}^{t}$ (see (2.5)), from (4.3) and (4.4) we have

$$
\dot{B}(t)=\mathscr{G}_{1}\left(H(t), \mathscr{H}^{t}, B(t)\right) \dot{H}(t)+\mathscr{G}_{2}\left(H(t), \mathscr{H}^{t}, B(t)\right)|\dot{H}(t)| .
$$

Since

$$
\operatorname{sgn}(\dot{H}(t))=\operatorname{sgn}\left(H(t)-\bar{H}_{1}\right)
$$

functions $\mathscr{G}_{1}$ and $\mathscr{G}_{2}$ depend on $\operatorname{sgn}(\dot{H}(t))$ through the difference $H(t)-\bar{H}_{1}$, too. Therefore, in order that the distinction between the addenda of the right-hand side of (4.5) makes sense, we assume $\mathscr{G}_{1}$ and $\mathscr{G}_{2}$ to be even functions of $H(t)-\bar{H}_{1}$. An equivalent formulation of (4.5) is

$$
\dot{B}(t)=\mu\left(H(t), \mathscr{H}^{t}, B(t)\right) \dot{H}(t),
$$

where

$$
\mu\left(H(t), \mathscr{H}^{t}, B(t)\right)=\mathscr{G}_{1}\left(H(t), \mathscr{H}^{t}, B(t)\right)+\mathscr{G}_{2}\left(H(t), \mathscr{H}^{t}, B(t)\right) \operatorname{sgn}\left(H(t)-\bar{H}_{1}\right) .
$$

Due to the scalar character of (4.6), the elimination of the parameter $t$ leads to

$$
\frac{\partial \tilde{B}}{\partial H}=\mu\left(H, \mathscr{H}^{t}, B\right),
$$

where, according to (2.5) and (4.2),

$$
B(t)=\tilde{\mathscr{B}}\left(H_{0}, H^{0}, B_{0}, P_{[0, t)}\right)=\tilde{B}\left(H(t), \mathscr{H}^{t}, H_{0}, B_{0}\right)
$$

is the solution of (4.1) expressed in terms of the intrinsic string $\mathscr{H}^{t}$.

Observe that $\mu$ is the sum of an even end an odd function with respect to $\left(H(t)-\bar{H}_{1}\right)$, namely,

$$
\mu\left(H(t), \mathscr{H}^{t}, B(t)\right)=\mathscr{G}^{(\mathrm{e})}\left(H(t), \mathscr{H}^{t}, B(t)\right)+\mathscr{G}^{(\mathrm{o})}\left(H(t), \mathscr{H}^{t}, B(t)\right)
$$

with

$$
\mathscr{G}^{(\mathrm{e})}\left(H(t), \mathscr{H}^{t}, B(t)\right)=\mathscr{G}_{1}\left(H(t), \mathscr{H}^{t}, B(t)\right)
$$




$$
\mathscr{G}^{(\mathrm{o})}\left(H(t), \mathscr{H}^{t}, B(t)\right)=\mathscr{G}_{2}\left(H(t), \mathscr{H}^{t}, B(t)\right) \operatorname{sgn}\left(H(t)-\bar{H}_{1}\right) .
$$

Finally, introducing the variation string

$$
\Delta \mathscr{H}^{t}=\left(H(t)-\bar{H}_{1}, \bar{H}_{1}-\bar{H}_{2}, \ldots, \bar{H}_{n-1}-\bar{H}_{n}, \ldots\right)
$$

and using the pair $\left(H(t), \Delta \mathscr{H}^{t}\right)$ instead of $\left(H(t), \mathscr{H}^{t}\right)$, an equivalent formulation of (4.5) is achieved, namely,

$$
\dot{B}(t)=\hat{\mu}\left(H(t), \Delta \mathscr{H}^{t}, B(t)\right) \dot{H}(t),
$$

where

$$
\begin{aligned}
& \hat{\mu}\left(H(t), \Delta \mathscr{H}^{t}, B(t)\right)=\hat{\mathscr{G}}^{(\mathrm{e})}\left(H(t), \Delta \mathscr{H}^{t}, B(t)\right)+\hat{\mathscr{G}}^{(\mathrm{o})}\left(H(t), \Delta \mathscr{H}^{t}, B(t)\right), \\
& \hat{\mathscr{G}}^{(\mathrm{e})}\left(H(t), \Delta \mathscr{H}^{t}, B(t)\right)=\mathscr{G}^{(\mathrm{e})}\left(H(t), \mathscr{H}^{t}, B(t)\right), \quad \hat{\mathscr{G}}^{(\mathrm{o})}\left(H(t), \Delta \mathscr{H}^{t}, B(t)\right)=\mathscr{G}^{(\mathrm{o})}\left(H(t), \mathscr{H}^{t}, B(t)\right) .
\end{aligned}
$$

The solution of (4.9) is then given by a functional of the variation string, namely,

$$
B(t)=\hat{B}\left(H(t), \Delta \mathscr{H}^{t}, H_{0}, B_{0}\right)
$$

A constitutive equation close to (4.9) and (4.10) is provided by the Praisach model and its extensions [14]. Nevertheless, in the Preisach model, the distinction between the true string and the reduced one is crucial [18].

\section{A simple history-differential model}

In this section, we discuss the simplest case of history-differential model supposing that all the "memory" of the magnet is resumed into the value of the magnetic field corresponding to the last inversion time, i.e., only $\bar{H}_{1}$ enter the definition of the magnetic state $m$. According to our initial assumption, we deal with magnetic histories belonging to $L B V$, so that, in a finite time interval, the inversion points are at most a numerable set. Therefore, letting $\bar{H}(t)=\bar{H}_{1}(t)$, the magnetic state $m$ is given by the triplet $m=(H, \bar{H}, B)$ and we can represent $\bar{H}(t)=H(\bar{t})$, where

$$
\bar{t}=\sup \left\{t_{0}<t: \operatorname{sgn}\left(\dot{H}\left(t_{0}^{+}\right)\right) \cdot \operatorname{sgn}\left(\dot{H}\left(t_{0}^{-}\right)\right) \leqslant 0\right\}
$$

The differential equation ruling the evolution of the magnetic induction is assumed to be

$$
\dot{B}(t)=\alpha \sigma(H(t)-\bar{H}(t))[f(H(t))-B(t)] \dot{H}(t)+g(H(t)) \dot{H}(t),
$$

where $\alpha>0$ and $\sigma, f, g$ fulfills the following assumptions: 
- $f: \mathbb{R} \rightarrow \mathbb{R}$ is a piecewise smooth, monotone increasing, odd function with

$$
0<a=\lim _{\xi \rightarrow \infty} f^{\prime}(\xi)<\infty
$$

- $g: \mathbb{R} \rightarrow \mathbb{R}^{+}$is a piecewise continuous even function satisfying

$$
\begin{aligned}
& g\left(\xi_{1}\right) \leqslant g\left(\xi_{2}\right) \quad \forall \xi_{1}, \xi_{2} \in \mathbb{R}:\left|\xi_{1}\right| \geqslant\left|\xi_{2}\right|, \\
& \lim _{\xi \rightarrow \infty} g(\xi)=\lim _{\xi \rightarrow \infty} f^{\prime}(\xi)=0, \\
& g(0)<f^{\prime}(0), \quad g(\xi) \leqslant f^{\prime}(\xi) \quad \forall \xi \in \mathbb{R} ;
\end{aligned}
$$

- $\sigma: \mathbb{R} \rightarrow \mathbb{R}$ is a smooth odd function such that

$$
\begin{aligned}
& \sigma(\xi)=-\sigma(-\xi)>0 \quad \forall \xi>0, \lim _{\xi \rightarrow \infty} \sigma(\xi)=1, \\
& -\xi \sigma^{\prime \prime}(\xi), \sigma^{\prime}(\xi) \geqslant 0 \quad \forall \xi \in \mathbb{R}, \sigma^{\prime}(0) \leqslant M<\infty .
\end{aligned}
$$

From (5.6) and (5.7), it follows that

$$
A(\xi):=\int_{0}^{\xi} \alpha \sigma(s) \mathrm{d} s
$$

is an even function with the following properties:

$$
A(\xi)=A(-\xi)>0, \quad \frac{1}{2} \alpha \sigma^{\prime}(\xi) \xi^{2} \leqslant A(\xi) \leqslant \frac{1}{2} \alpha \sigma^{\prime}(0) \xi^{2} \forall \xi \in \mathbb{R} .
$$

Eq. (5.1) enters the class of models described by (4.6) letting

$$
\mathscr{G}^{(\mathrm{e})}=\tilde{\mathscr{G}}^{(\mathrm{e})}(H, H-\bar{H})=g(H), \quad \mathscr{G}^{(\mathrm{o})}=\tilde{\mathscr{G}}^{(\mathrm{o})}(H, H-\bar{H}, B)=\alpha \sigma(H-\bar{H})(f(H)-B) .
$$

Therefore, the elimination of the parameter $t$ in (5.1) leads to the rate-independent equation

$$
\frac{\partial \tilde{B}}{\partial H}=\alpha \sigma(H-\bar{H})[f(H)-B]+g(H),
$$

whose solution determines a path in the $(H, B)$-plane.

Remark 5.1. Since the magnetic state $m$ is given by the triplet $(H, \bar{H}, B)$, there are infinitely many paths passing through each point of the $(H, B)$-plane. This property is typical of any history type model. In Madelung's model, on the contrary, each point lies just on two solution-curves: one 
related to $\dot{H}>0$ (loading curve), and the other to $\dot{H}<0$ (unloading curve). Any solution $\tilde{B}\left(H, \bar{H} ; H_{i}, B_{i}\right)$ of $(5.10)$ may represent either a loading or an unloading branch. In particular, if $H>\bar{H}$ and $H_{i} \geqslant \bar{H}$ then $\tilde{B}\left(H, \bar{H} ; H_{i}, B_{i}\right)$ takes the meaning of loading curve; on the contrary, for $H<\bar{H}$ and $H_{i} \leqslant \bar{H}$, it represents an unloading curve. All other relations between $H, H_{i}$ and $\bar{H}$ are physically meaningless.

A straightforward calculation yields the solutions of (5.10) starting from the point $\left(H_{i}, B_{i}\right)$ of the $(H, B)$-plane, when $\bar{H}$ is kept constant:

$$
\tilde{B}\left(H, \bar{H}, H_{i}, B_{i}\right)=f(H)+\left[\left[B_{i}-f(\bar{H})\right] \mathrm{e}^{A\left(H_{i}-\bar{H}\right)}-\int_{H_{i}}^{H}\left[f^{\prime}(\eta)-g(\eta)\right] \mathrm{e}^{A(\eta-\bar{H})} \mathrm{d} \eta\right] \mathrm{e}^{-A(H-\bar{H})}
$$

As the function $\bar{H}(t)$ changes its value at the inversion points only, the easiest method to find the general solution of (5.10) is to perform repeated integrations over each branch between two consecutive inversion points. To do this we identify the initial data of each branch with the inversion point reached by the previous one. Therefore, it is of particular interest to construct the maximal branches, i.e., solutions (5.11) with $H_{i}=\bar{H}$ :

$$
\tilde{B}\left(H, \bar{H} ; \bar{H}, B_{i}\right)=f(H)+\left[\left[B_{i}-f(\bar{H})\right]-\int_{\bar{H}}^{H}\left[f^{\prime}(\eta)-g(\eta)\right] \mathrm{e}^{A(\eta-\bar{H})} \mathrm{d} \eta\right] \mathrm{e}^{-A(H-\bar{H})} .
$$

Taking the partial derivative of (5.12) with respect to $H$, we obtain the slope of the branch

$$
\frac{\partial \tilde{B}}{\partial H}=\alpha \sigma(H-\bar{H})\left[\int_{\bar{H}}^{H}\left[f^{\prime}(\eta)-g(\eta)\right] \mathrm{e}^{A(\eta-\bar{H})} \mathrm{d} \eta-\left[B_{i}-f(\bar{H})\right]\right] \mathrm{e}^{-A(H-\bar{H})}+g(H) .
$$

At every point $H$ where $g$ is differentiable, a further derivation of (5.13) provides the curvature

$$
\begin{aligned}
\frac{\partial^{2} \tilde{B}}{\partial H^{2}}= & \left(\alpha \sigma^{\prime}(H-\bar{H})-\alpha^{2} \sigma^{2}(H-\bar{H})\right)\left[\int_{\bar{H}}^{H}\left[f^{\prime}(\eta)-g(\eta)\right] \mathrm{e}^{A(\eta-\bar{H})} \mathrm{d} \eta-\left[B_{i}\right.\right. \\
& -f(\bar{H})]] \mathrm{e}^{-A(H-\bar{H})}+\alpha \sigma(H-\bar{H})\left[f^{\prime}(H)-g(H)\right]+g^{\prime}(H) .
\end{aligned}
$$

In particular, at the beginning of each branch (i.e., for $H=\bar{H}$ ) we have

$$
\left.\frac{\partial \tilde{B}}{\partial H}\right|_{H=\bar{H}}=g(\bar{H}),\left.\quad \frac{\partial^{2} \tilde{B}}{\partial H^{2}}\right|_{H=\bar{H}}=-\alpha \sigma^{\prime}(0)\left[B_{i}-f(\bar{H})\right]+g^{\prime}(\bar{H}),
$$

where the latter equality holds only if $g^{\prime}(H)$ is well defined at $H=\bar{H}$.

Assuming the hysteretic element starts from the virgin state $m_{\mathrm{v}}=(0,0,0)$, namely, from the initial data 


$$
B_{i}=0, \quad \bar{H}=H_{i}=0,
$$

then (5.12) yields the initial magnetisation curve

$$
\tilde{B}(H, 0 ; 0,0)=f(H)-\left[\int_{0}^{H}\left[f^{\prime}(\eta)-g(\eta)\right] \mathrm{e}^{A(\eta)} \mathrm{d} \eta\right] \mathrm{e}^{-A(H)} .
$$

At the beginning such a curve has an increasing slope and a oblique flexus. In fact, from (5.14) we have

$$
\left.\frac{\partial \tilde{B}}{\partial H}\right|_{H=0}=g(0)>0,\left.\quad \frac{\partial^{2} \tilde{B}}{\partial H^{2}}\right|_{H=0}=g^{\prime}(0)=0 .
$$

The last equality requires $g$ to be smooth enough and follows since $g$ is even. A Taylor expansion of (5.14) at $H=0$, with null initial data, provides

$$
\frac{\partial^{2} \tilde{B}}{\partial H^{2}} \approx 2 \alpha \sigma^{\prime}(0)\left[f^{\prime}(0)-g(0)\right] H
$$

Thus, by virtue of (5.5), we argue that near the origin the second derivative with respect to $H$ of the initial magnetisation curve has the same sign of $H$.

Assumption (5.4) implies that every magnetisation curve given by (5.12) (and in particular the initial one) approaches $f(H)$ as $H \rightarrow \infty$. In fact

$$
\lim _{H \rightarrow \infty} \tilde{B}\left(H, \bar{H} ; \bar{H}, B_{i}\right)-f(H)=\lim _{H \rightarrow \infty}\left[\left[B_{i}-f(\bar{H})\right]-\int_{\bar{H}}^{H}\left[f^{\prime}(\eta)-g(\eta)\right] \mathrm{e}^{A(\eta-\bar{H})} \mathrm{d} \eta\right] \mathrm{e}^{-A(H-\bar{H})}=0 .
$$

Remark 5.2. The integral inside the squared brackets in (5.16) diverges for large $H$, so that all curves described by (5.12) approach $f(H)$ from below as $H \rightarrow \infty$. Analogously, the same curves approach $f(H)$ from above as $H \rightarrow-\infty$. If $|H|$ is so large that $B(H, \bar{H})$ is indistinguishable from $f(H)$ (and so it remains when $|H|$ increases), then we say that the material is "loaded to saturation".

By virtue of (5.2)-(5.4), we take the limit in (5.12) according to (5.16) and obtain the curves with inversion point $\bar{H}$ at infinity:

$$
\begin{aligned}
& B_{+\infty}(H)=\lim _{\bar{H} \rightarrow+\infty} \tilde{B}(H, \bar{H} ; \bar{H}, f(\bar{H}))=f(H)+\int_{H}^{\infty}\left[f^{\prime}(\eta)-g(\eta)\right] \mathrm{e}^{-\alpha \eta} \mathrm{d} \eta \mathrm{e}^{\alpha H}, \\
& B_{-\infty}(H)=\lim _{\bar{H} \rightarrow-\infty} \tilde{B}(H, \bar{H} ; \bar{H}, f(\bar{H}))=f(H)-\int_{-\infty}^{H}\left[f^{\prime}(\eta)-g(\eta)\right] \mathrm{e}^{\alpha \eta} \mathrm{d} \eta \mathrm{e}^{-\alpha H} .
\end{aligned}
$$


As we shall see later (see Section 5.1), (5.17) and (5.18) provide the upper and lower branches of the major hysteresis loop, so that all hysteresis paths are localised into the region

$$
\mathscr{M}=\left\{(H, B) \in \mathbb{R}^{2}: B_{-\infty}(H) \leqslant B \leqslant B_{+\infty}(H)\right\} .
$$

Moreover, the residual magnetisation (or retentivity) $B_{\mathrm{r}}$ can be easily evaluated as

$$
B_{\mathrm{r}}=B_{+\infty}(0)=\int_{0}^{\infty}\left[f^{\prime}(\eta)-g(\eta)\right] \mathrm{e}^{-\alpha \eta} \mathrm{d} \eta .
$$

On the other hand, the coercive magnetic field (or coercivity) $H_{\mathrm{c}}$ is implicitly given by $B_{-\infty}\left(H_{\mathrm{c}}\right)=0$, namely,

$$
f\left(H_{\mathrm{c}}\right)=\int_{-\infty}^{H_{\mathrm{c}}}\left[f^{\prime}(\eta)-g(\eta)\right] \mathrm{e}^{\alpha \eta} \mathrm{d} \eta \mathrm{e}^{-\alpha H_{\mathrm{c}}} .
$$

Finally, we stress that constitutive functions in (3.2)-(3.7) are required to comply the requirement of piecewise monotonicity, as stated in the following:

Remark 5.3. In (3.2)-(3.7) $\alpha, f, g$ and $\sigma$ have to be chosen so that the slope $\partial \tilde{B} / \partial H$ of the path is always non-negative. From (5.10) this means

$$
\alpha \sigma(H-\bar{H})[f(H)-B]+g(H) \geqslant 0 \quad \forall(H, \bar{H}, B) .
$$

By virtue of (5.2)-(5.7), it is easy to check the following sufficient condition:

$$
\alpha \max \left\{\left|f(H)-B_{+\infty}(H)\right|,\left|f(H)-B_{-\infty}(H)\right|\right\} \leqslant g(H) .
$$

This is a severe restriction which makes the mathematical model applicable to ferromagnetic materials with narrow hysteresis loops, as well as isoperms, only.

\subsection{A necessary improvement}

The model described by (5.1) has a fault: not all the curves representing solutions of (5.11) and starting inside the region $\mathscr{M}$ are entirely contained in $\mathscr{M}$.

In order to see that, let us consider a loading curve with initial state $m_{i}=\left(H_{i}, \bar{H}=H_{i}, B_{i}\right)$ such that $\left(H_{i}, B_{i}\right)$ is very close to the curve $B_{-\infty}(H)$, for instance, $B_{i}=B_{-\infty}\left(H_{i}\right)+\delta, \delta \ll 1$. Observe that the slope of $B_{-\infty}$ at $H=H_{i}$ is given by

$$
B_{-\infty}^{\prime}\left(H_{i}\right)=\alpha\left[f\left(H_{i}\right)-B_{-\infty}\left(H_{i}\right)\right]+g\left(H_{i}\right),
$$

whereas the slope of the curve starting from $m_{i}$ is

$$
\frac{\partial B}{\partial H}\left(H_{i}\right)=g\left(H_{i}\right)<B_{-\infty}^{\prime}\left(H_{i}\right)
$$

therefore such a curve will cross $B_{-\infty}(H)$ and leave $\mathscr{M}$. 
In order to avoid such a problem we modify constitutive equation (5.1) as

$$
\dot{B}(t)=\alpha \sigma_{1}(H(t), \bar{H}(t), B(t))[f(H(t))-B(t)] \dot{H}(t)+g(H(t)) \dot{H}(t),
$$

where

$$
\sigma_{1}(H(t), \bar{H}(t), B(t))= \begin{cases}\sigma_{1}(H(t)-\bar{H}(t)) & \text { for } B_{-\infty}(H(t))<B(t)<B_{+\infty}(H(t)), \\ 1 & \text { for } B(t)=B_{-\infty}(H(t)), \\ -1 & \text { for } B(t)=B_{+\infty}(H(t)) .\end{cases}
$$

The structure of (5.21) ensures that (5.20) describes the same behaviour of (5.1) inside $\mathscr{M}$, but when a curve reaches one of the extremal curves $B_{-\infty}$ or $B_{+\infty}$, then the representative point moves on this latter curve up to a turning point.

It is easy to see that a $B_{-\infty}$ may be reached just by loading curves as well as $B_{+\infty}$ may be crossed just by unloading curves.

\section{Some general properties}

In order to emphasise some relevant characteristics of this model, we take a particular choice of the constitutive functions. We consider $f$ and $g$ defined as in (3.7), namely,

$$
f(H)=\left\{\begin{array}{ll}
a\left[H+H^{*}\right]-b H^{*} & \text { for } H<-H^{*}, \\
b H & \text { for }-H^{*} \leqslant H \leqslant H^{*}, \\
a\left[H-H^{*}\right]+b H^{*} & \text { for } H>H^{*},
\end{array} \quad g(H)= \begin{cases}u & \text { for }|H| \leqslant H^{*}, \\
a & \text { for }|H|>H^{*},\end{cases}\right.
$$

where

$$
b>u>a>0 .
$$

Under these assumptions (5.10), as well as (5.20) inside $\mathscr{M}$, yields the following differential system:

$$
\frac{\partial \tilde{B}}{\partial H}= \begin{cases}\alpha \sigma(H-\bar{H})\left(a H+H^{*}(a-b)-B\right)+a, & H<-H^{*} \\ \alpha \sigma(H-\bar{H})(b H-B)+u, & |H| \leqslant H^{*} \\ \alpha \sigma(H-\bar{H})\left(a H-H^{*}(a-b)-B\right)+a, & H>H^{*}\end{cases}
$$

Letting $\alpha=\lim _{\xi \rightarrow+\infty} \alpha \sigma(\xi)$, the function $\alpha \operatorname{sgn}(\dot{H})=\alpha \operatorname{sgn}(H-\bar{H})$ can be viewed as the limiting case of $\alpha \sigma(H-\bar{H})$ as $|H-\bar{H}| \rightarrow \infty$. So the behaviour of this model approaches that of Coleman and Hodgdon's model (3.5)-(3.7) as $|H-\bar{H}| \rightarrow \infty$.

For any given initial condition $\left(H_{i}, B_{i}\right)$ which lies inside the region

$$
\mathscr{M}^{*}=\left\{(H, B) \in \mathscr{M}:|H| \leqslant H^{*}\right\},
$$


the solution $B(H, \bar{H})=\tilde{B}\left(H, \bar{H}, H_{i}, B_{i}\right)$ of $(6.3)$ is given by

$$
B(H, \bar{H})= \begin{cases}a H+H^{*}(a-b)+\mathrm{e}^{-D(\bar{H}, H)}\left(C-\int_{\bar{H}}^{-H^{*}}[b-u] \mathrm{e}^{D(\bar{H}, \eta)} \mathrm{d} \eta\right), & H<-H^{*}, \\ b H+\mathrm{e}^{-D(\bar{H}, H)}\left(C-\int_{\bar{H}}^{H}(b-u) \mathrm{e}^{D(\bar{H}, \eta)} \mathrm{d} \eta\right), & |H| \leqslant H^{*}, \\ a H-H^{*}(a-b)+\mathrm{e}^{-D(\bar{H}, H)}\left(C-\int_{\bar{H}}^{H^{*}}[b-u] \mathrm{e}^{D(\bar{H}, \eta)} \mathrm{d} \eta\right), & H>H^{*},\end{cases}
$$

where

$$
\begin{aligned}
& C\left(\bar{H}, H_{i}, B_{i}\right)=\left(B_{i}-b H_{i}\right) \mathrm{e}^{D\left(\bar{H}, H_{i}\right)}+\int_{\bar{H}}^{H_{i}}[b-u] \mathrm{e}^{D(\bar{H}, \eta)} \mathrm{d} \eta \\
& D(\xi, \eta)=A(\eta-\xi)-A(\xi) .
\end{aligned}
$$

The solution (6.4) of (6.3) is constructed by assuming $H_{i} \in\left[-H^{*}, H^{*}\right]$, so that $C$ is the constant of integration of $(6.3)_{2}$. The solutions $(6.4)_{1}$ and $(6.4)_{3}$ of $(6.3)_{1}$ and $(6.3)_{3}$ are obtained by assuming the continuity of $B(H, \bar{H})$, respectively, at $H=-H^{*}$ and $H=H^{*}$. However, assumptions different from $H_{i} \in\left[-H^{*}, H^{*}\right]$ would change the relation between $C$ and the initial point of integration $\left(H_{i}, B_{i}\right)$, but not the general form (6.4) of the solution.

When $H \geqslant \bar{H}$, the curve $B(H, \bar{H})$ takes the meaning of loading curve, and it is denoted by $B_{\mathrm{L}}(H, \bar{H})$ ). On the other hand, it represents an unloading curve, denoted by $B_{\mathrm{U}}(H, \bar{H})$, for $H \leqslant \bar{H}$. Moreover, in view of (6.4), if $H_{i} \geqslant \bar{H}$, then the curve must be considered as the branch $B_{\mathrm{L}}(H, \bar{H})$, $H \geqslant \bar{H}$. In fact, in this case only the part of the path with $H>\bar{H}$ makes sense. Accordingly, if $H_{i} \leqslant \bar{H}$ just the branch $B_{\mathrm{U}}(H, \bar{H})(H \leqslant \bar{H})$ must be considered.

The asymptotic behaviour of $B_{\mathrm{L}}(H, \bar{H})$ as $H \rightarrow \infty$, as well as the behaviour of $B_{\mathrm{U}}(H, \bar{H})$ as $H \rightarrow-\infty$, are independent of $\bar{H}$. In particular, it is easy to check that $B_{\mathrm{L}}(H, \bar{H})$ approaches the line $B=a H-H^{*}(a-b)$ from below as $H \rightarrow \infty$, and $B_{\mathrm{U}}(H, \bar{H})$ approaches the line $B=a H+H^{*}(a-b)$ from above as $H \rightarrow-\infty$. Therefore, a necessary condition for $(H, B)$ to lie inside the region $\mathscr{M}$ of the major loop is given by

$$
a H+H^{*}(a-b) \leqslant B \leqslant a H-H^{*}(a-b) .
$$

This condition is satisfied by any solution whose initial data $\left(H_{i}, B_{i}\right)$ do the same.

Consider now the unloading curves passing through the point $\left(H_{i}, B_{i}\right)=\left(H^{*}, b H^{*}\right)$. From (6.5) we have

$$
C\left(\bar{H}, H^{*}, b H^{*}\right)=(b-u) \int_{\bar{H}}^{H^{*}} \mathrm{e}^{D(\bar{H}, \eta)} \mathrm{d} \eta
$$

and

$$
B_{\mathrm{L}}(H, \bar{H})= \begin{cases}a H+H^{*}(a-b)+(b-u) \mathrm{e}^{-D(\bar{H}, H)} \int_{-H^{*}}^{H^{*}} \mathrm{e}^{D(\bar{H}, \eta)} \mathrm{d} \eta, & H<-H^{*}, \\ b H+(b-u) \mathrm{e}^{-D(\bar{H}, H)} \int_{H}^{H^{*}} \mathrm{e}^{D(\bar{H}, \eta)} \mathrm{d} \eta, & |H| \leqslant H^{*} \\ a H-H^{*}(a-b), & H>H^{*}\end{cases}
$$


On the other hand, if $\left(H_{i}, B_{i}\right)=\left(-H^{*},-b H^{*}\right)$, we have

$$
\begin{aligned}
& C\left(\bar{H},-H^{*},-b H^{*}\right)=(b-u) \int_{\bar{H}}^{-H^{*}} \mathrm{e}^{D(\bar{H}, \eta)} \mathrm{d} \eta, \\
& B_{\mathrm{U}}(H, \bar{H})= \begin{cases}a H+H^{*}(a-b), & H<-H^{*}, \\
b H-(b-u) \mathrm{e}^{-D(\bar{H}, H)} \int_{-H^{*}}^{H} \mathrm{e}^{D(\bar{H}, \eta)} \mathrm{d} \eta, & |H| \leqslant H^{*}, \\
a H-H^{*}(a-b)-(b-u) \mathrm{e}^{-D(\bar{H}, H)} \int_{-H^{*}}^{H^{*}} \mathrm{e}^{D(\bar{H}, \eta)} \mathrm{d} \eta, & H>H^{*}\end{cases}
\end{aligned}
$$

Both branches of the major loop can be easily evaluated. Reminding that

$$
A(\bar{H}-H)-A(\bar{H})=-\alpha \sigma\left(\xi^{*}\right) H
$$

choosing a suitable $\xi^{*} \in[\bar{H}-H, H]$, we have

$$
\lim _{\bar{H} \rightarrow \pm \infty} D(\bar{H}, H)=\lim _{\bar{H} \rightarrow \pm \infty} A(\bar{H}-H)-A(\bar{H})=\mp \alpha H
$$

Since the upper and lower branch of the major hysteresis loop are given by the asymptotical loading and the loading curves $B_{-\infty}=\lim _{\bar{H} \rightarrow-\infty} B_{\mathrm{L}}(H, \bar{H}), B_{+\infty}=\lim _{\bar{H} \rightarrow+\infty} B_{\mathrm{U}}(H, \bar{H})$, from (6.8) and (6.9) we have

$$
B_{-\infty}(H)= \begin{cases}a H+H^{*}(a-b), & H<-H^{*} \\ b H-((b-u) / \alpha)\left(1-\mathrm{e}^{-\alpha\left(H+H^{*}\right)}\right), & |H| \leqslant H^{*} \\ a H-H^{*}(a-b)-((b-u) / \alpha) \mathrm{e}^{-\alpha H}\left(\mathrm{e}^{\alpha H^{*}}-\mathrm{e}^{-\alpha H^{*}}\right), & H>H^{*}\end{cases}
$$

and

$$
B_{+\infty}(H)= \begin{cases}a H+H^{*}(a-b)+((b-u) / \alpha) \mathrm{e}^{\alpha H}\left(\mathrm{e}^{\alpha H^{*}}-\mathrm{e}^{-\alpha H^{*}}\right), & H<-H^{*} \\ b H+((b-u) / \alpha)\left(1-\mathrm{e}^{\alpha\left(H-H^{*}\right.}\right), & |H| \leqslant H^{*} \\ a H-H^{*}(a-b), & H>H^{*}\end{cases}
$$

As a consequence, the residual magnetisation $B_{\mathrm{r}}$ is given by

$$
B_{\mathrm{r}}=B_{+\infty}(0)=\frac{(b-u)}{\alpha}\left(1-\mathrm{e}^{-\alpha H^{*}}\right)
$$

and the coercive magnetic field $H_{\mathrm{c}}$ is the value that solves the equation

$$
\begin{cases}b H_{\mathrm{c}}-((b-u) / \alpha)\left(1-\mathrm{e}^{-\alpha\left(H_{\mathrm{c}}+H^{*}\right)}\right)=0 & \text { if } 0<H_{\mathrm{c}} \leqslant H^{*} \\ a H_{\mathrm{c}}-H^{*}(a-b)-((b-u) / \alpha) \mathrm{e}^{-\alpha H_{\mathrm{c}}}\left(\mathrm{e}^{\alpha H^{*}}-\mathrm{e}^{-\alpha H^{*}}\right)=0 & \text { if } H_{\mathrm{c}}>H^{*}\end{cases}
$$

Taking the limits of (6.4) as $\bar{H} \rightarrow \pm \infty$ for any given initial data $\left(H_{i}, B_{i}\right) \in \mathscr{M}$ we have, respectively, 


$$
\begin{aligned}
& C_{\infty}\left(H_{i}, B_{i}\right)=\left(B_{i}-b H_{i}\right) \mathrm{e}^{-\alpha H_{i}}-(b-u) \int_{H_{i}}^{\infty} \mathrm{e}^{-\alpha s} \mathrm{~d} \eta=\mathrm{e}^{-\alpha H_{i}}\left(B_{i}-b H_{i}-\frac{(b-u)}{\alpha}\right), \\
& C_{-\infty}\left(H_{i}, B_{i}\right)=\left(B_{i}-b H_{i}\right) \mathrm{e}^{\alpha H_{i}}+(b-u) \int_{-\infty}^{H_{i}} \mathrm{e}^{\alpha s} \mathrm{~d} \eta=\mathrm{e}^{\alpha H_{i}}\left(B_{i}-b H_{i}+\frac{(b-u)}{\alpha}\right)
\end{aligned}
$$

and the general expressions for the loading and unloading branches passing through $\left(H_{i}, B_{i}\right)$ and having an inversion point at infinity are, respectively,

$$
\begin{aligned}
& B_{\mathrm{L}}(H,-\infty)= \begin{cases}a H+H^{*}(a-b)+\mathrm{e}^{-\alpha H}\left(C_{-\infty}-((b-u) / \alpha) \mathrm{e}^{-\alpha H^{*}}\right), & H<-H^{*}, \\
b H+\left(C_{-\infty} \mathrm{e}^{-\alpha H}-((b-u) / \alpha)\right), & |H| \leqslant H^{*}, \\
a H-H^{*}(a-b)+\mathrm{e}^{-\alpha H}\left(C_{-\infty}-((b-u) / \alpha) \mathrm{e}^{\alpha H^{*}}\right), & H>H^{*},\end{cases} \\
& B_{\mathrm{U}}(H, \infty)= \begin{cases}a H+H^{*}(a-b)+\mathrm{e}^{\alpha H}\left(C_{\infty}+((b-u) / \alpha) \mathrm{e}^{\alpha H^{*}}\right), & H<-H^{*}, \\
b H+\left(C_{\infty} \mathrm{e}^{\alpha H}+((b-u) / \alpha)\right), & |H| \leqslant H^{*}, \\
a H-H^{*}(a-b)+\mathrm{e}^{\alpha H}\left(C_{\infty}+((b-u) / \alpha) \mathrm{e}^{-\alpha H^{*}}\right), & H>H^{*}\end{cases}
\end{aligned}
$$

Observe that (6.17) and (6.18) are the same as the lower and upper branch (3.8) and (3.9) of the major hysteresis loop derived from Coleman and Hodgdon's model.

\subsection{A further refinement}

We stress that constitutive equations previously considered yield a major hysteresis loop whose shape depends on the slope of the initial magnetisation curve. Unfortunately, this might be a severe limitation in fitting the model with experimental data. In order to fix this problem, (6.1) can be easily modified by substituting $g(H)$ with a function $\gamma(H, H-\bar{H})$ defined as

$$
\gamma(\eta, \eta-\xi)=\left\{\begin{array}{ll}
\gamma^{*}(\eta-\xi) & \text { for }|\eta| \leqslant H^{*}, \\
a & \text { for }|\eta|>H^{*},
\end{array} \quad \gamma^{*}(\zeta)= \begin{cases}u & \text { for }|\zeta| \leqslant 2 H^{*} \\
v(\zeta) & \text { for }|\zeta|>2 H^{*}\end{cases}\right.
$$

where $v: \mathbb{R} \rightarrow \mathbb{R}$ is a smooth even function such that

$$
v(\xi) \leqslant u, \quad v^{\prime}(\xi)<0 \forall \xi>2 H^{*}, \quad \lim _{\xi \rightarrow \infty} v(\xi)=a .
$$

As a consequence (6.3) becomes

$$
\frac{\partial \tilde{B}}{\partial H}= \begin{cases}\alpha \sigma(H-\bar{H})\left(a H+H^{*}(a-b)-B\right)+a, & H<-H^{*} \\ \alpha \sigma(H-\bar{H})(b H-B)+\gamma^{*}(H-\bar{H}), & |H| \leqslant H^{*} \\ \alpha \sigma(H-\bar{H})\left(a H-H^{*}(a-b)-B\right)+a, & H>H^{*}\end{cases}
$$


and every solution $B(H, \bar{H})=\tilde{B}\left(H, \bar{H}, H_{i}, B_{i}\right)$ of $(6.20)$ is given by

$$
B(H, \bar{H})= \begin{cases}a H+H^{*}(a-b)+\mathrm{e}^{-D(\bar{H}, H)}\left(C-\int_{\bar{H}}^{-H^{*}}\left[b-\gamma^{*}(\eta-\bar{H})\right] \mathrm{e}^{D(\bar{H}, \eta)} \mathrm{d} \eta\right), & H<-H^{*}, \\ b H+\mathrm{e}^{-D(\bar{H}, H)}\left(C-\int_{\bar{H}}^{H}\left[b-\gamma^{*}(\eta-\bar{H})\right] \mathrm{e}^{D(\bar{H}, \eta)} \mathrm{d} \eta\right), & |H| \leqslant H^{*}, \\ a H-H^{*}(a-b)+\mathrm{e}^{-D(\bar{H}, H)}\left(C-\int_{\bar{H}}^{H^{*}}\left[b-\gamma^{*}(\eta-\bar{H})\right] \mathrm{e}^{D(\bar{H}, \eta)} \mathrm{d} \eta\right), & H>H^{*},\end{cases}
$$

where

$$
\begin{aligned}
& C\left(\bar{H}, H_{i}, B_{i}\right)=\left(B_{i}-b H_{i}\right) \mathrm{e}^{D\left(\bar{H}, H_{i}\right)}+\int_{\bar{H}}^{H_{i}}\left[b-\gamma^{*}(\eta-\bar{H})\right] \mathrm{e}^{D(\bar{H}, \eta)} \mathrm{d} \eta \\
& D(\xi, \eta)=A(\eta-\xi)-A(\xi) .
\end{aligned}
$$

Expression (6.21) is constructed by solving (6.20) 2 and assuming $H_{i} \in\left[-H^{*}, H^{*}\right]$, so that $C$ is the constant of integration. The solutions of $(6.20)_{1}$ and $(6.20)_{3}$ are obtained by assuming the continuity of $B(H, \bar{H})$, respectively, at $H=-H^{*}$ and $H=H^{*}$. Nevertheless, assumptions different from $H_{i} \in\left[-H^{*}, H^{*}\right]$ would change the relation between $C$ and the initial point of integration $\left(H_{i}, B_{i}\right)$, but not the general form (6.21) of the solution.

Taking the limits of (6.21) as $\bar{H} \rightarrow \pm \infty$ for any given initial data $\left(H_{i}, B_{i}\right) \in \mathscr{M}$ we have, respectively,

$$
\begin{aligned}
& C_{\infty}\left(H_{i}, B_{i}\right)=\left(B_{i}-b H_{i}\right) \mathrm{e}^{-\alpha H_{i}}-(b-a) \int_{H_{i}}^{\infty} \mathrm{e}^{-\alpha s} \mathrm{~d} \eta=\mathrm{e}^{-\alpha H_{i}}\left(B_{i}-b H_{i}-((b-a) / \alpha)\right) \\
& C_{-\infty}\left(H_{i}, B_{i}\right)=\left(B_{i}-b H_{i}\right) \mathrm{e}^{\alpha H_{i}}+(b-a) \int_{-\infty}^{H_{i}} \mathrm{e}^{\alpha s} \mathrm{~d} \eta=\mathrm{e}^{\alpha H_{i}}\left(B_{i}-b H_{i}+((b-a) / \alpha)\right)
\end{aligned}
$$

and the general expressions for the loading and unloading branches passing through $\left(H_{i}, B_{i}\right)$ and having an inversion point at infinity are, respectively,

$$
\begin{aligned}
& B_{\mathrm{L}}(H,-\infty)= \begin{cases}a H+H^{*}(a-b)+\mathrm{e}^{-\alpha H}\left(C_{-\infty}-((b-a) / \alpha) \mathrm{e}^{-\alpha H^{*}}\right), & H<-H^{*}, \\
b H+\left(C_{-\infty} \mathrm{e}^{-\alpha H}-((b-a) / \alpha)\right), & |H| \leqslant H^{*}, \\
a H-H^{*}(a-b)+\mathrm{e}^{-\alpha H}\left(C_{-\infty}-((b-a) / \alpha) \mathrm{e}^{\alpha H^{*}}\right), & H>H^{*},\end{cases} \\
& B_{\mathrm{U}}(H, \infty)= \begin{cases}a H+H^{*}(a-b)+\mathrm{e}^{\alpha H}\left(C_{\infty}+((b-a) / \alpha) \mathrm{e}^{\alpha H^{*}}\right), & H<-H^{*}, \\
b H+\left(C_{\infty} \mathrm{e}^{\alpha H}+((b-a) / \alpha)\right), & |H| \leqslant H^{*}, \\
a H-H^{*}(a-b)+\mathrm{e}^{\alpha H}\left(C_{\infty}+((b-a) / \alpha) \mathrm{e}^{-\alpha H^{*}}\right), & H>H^{*} .\end{cases}
\end{aligned}
$$


In particular, the major hysteresis loop is given by the curves

$$
B_{-\infty}(H)= \begin{cases}a H+H^{*}(a-b), & H<-H^{*} \\ b H-((b-a) / \alpha)\left(1-\mathrm{e}^{-\alpha\left(H+H^{*}\right)}\right), & |H| \leqslant H^{*} \\ a H-H^{*}(a-b)-((b-a) / \alpha) \mathrm{e}^{-\alpha H}\left(\mathrm{e}^{\alpha H^{*}}-\mathrm{e}^{-\alpha H^{*}}\right), & H>H^{*}\end{cases}
$$

and

$$
B_{+\infty}(H)= \begin{cases}a H+H^{*}(a-b)+((b-a) / \alpha) \mathrm{e}^{\alpha H}\left(\mathrm{e}^{\alpha H^{*}}-\mathrm{e}^{-\alpha H^{*}}\right), & H<-H^{*} \\ b H+((b-a) / \alpha)\left(1-\mathrm{e}^{\alpha\left(H-H^{*}\right)}\right), & |H| \leqslant H^{*} \\ a H-H^{*}(a-b), & H>H^{*}\end{cases}
$$

We stress that (6.26) and (6.27) differ from previous expressions (3.8) and (3.9) of Coleman and Hodgdon's model and from (6.17) and (6.18). In fact, here the factor $(b-a)$ is present instead of $(b-u)$. As a consequence, the shape of the major hysteresis loop (6.28) and (6.29) is independent of $u$, the initial slope of the first magnetisation curve.

\section{Long time asymptotic properties}

In this section, we scrutinise long time behaviour and periodic response of the model. In particular, we show the following properties:

(a) there exist limiting loops corresponding to oscillatory precesses;

(b) there exists a universal (i.e., independent of the initial state) process of demagnetisation;

(c) all stationary magnetic states are stable in the $C^{0}$-norm with respect to perturbations $H$ of bounded variation.

Both (a) and (b) are as in the Coleman-Hodgdon model, but (c) is peculiar of the present setting and prevents the model to exhibit self-magnetisation.

Henceforth, in order to simplify the procedure of the proofs, we confine our attention to processes that lie in the region $\mathscr{M}^{*}=\left\{(H, B) \in \mathscr{M}: H \in\left[-H^{*}, H^{*}\right]\right\}$ of the $(H, B)$-plane. This choice is not too restrictive, in that the major-loop area is mainly contained in $\mathscr{M}^{*}$, as pointed out in the previous section. To this end, we keep $H, \bar{H} \in\left[-H^{*}, H^{*}\right]$. As a consequence $g(H)=u$ and the evolution equation (5.10) reduces to

$$
\frac{\partial B}{\partial H}(H, \bar{H})=\alpha \sigma(H-\bar{H})(b H-B)+u .
$$

Note that, even if the modification of Section 6.1 is considered, the evolution equation reduces to (7.1). In fact, if $H, \bar{H} \in\left[-H^{*}, H^{*}\right]$, we have $\gamma(H, H-\bar{H})=\gamma^{*}(H-\bar{H})=u$.

Integrating (7.1) over a process that does not exhibit inversion points ( $\bar{H}$ is kept constant), the solution is

$$
B(H, \bar{H})=b H+\mathrm{e}^{-A(H-\bar{H})}\left(C^{*}-(b-u) \int_{0}^{H-\bar{H}} \mathrm{e}^{A(\eta)} \mathrm{d} \eta\right)
$$


where $C^{*}$ depends on $B_{i}, H_{i}$ and $\bar{H}$ as follows:

$$
C^{*}\left(\bar{H}, H_{i}, B_{i}\right)=\left(B_{i}-b H_{i}\right) \mathrm{e}^{A\left(H_{i}-\bar{H}\right)}+(b-u) \int_{0}^{H_{i}-\bar{H}} \mathrm{e}^{A(\eta)} \mathrm{d} \eta .
$$

Thereby, assuming

$$
I(\xi)=(b-u) \int_{0}^{\xi} \mathrm{e}^{A(\eta)} \mathrm{d} \eta \quad \text { and } \quad \Delta H=|H-\bar{H}|,
$$

Eq. (7.2) can be rewritten as

$$
B(H, \bar{H})=b H+\mathrm{e}^{-A(\Delta H)}\left[\left(B_{i}-b H_{i}\right) \mathrm{e}^{A\left(H_{i}-\bar{H}\right)}+I\left(H_{i}-\bar{H}\right)-\operatorname{sgn}(H-\bar{H}) I(\Delta H)\right] .
$$

\subsection{Limiting loops}

The limiting-loop property of the ferromagnetic material describes a well-known phenomenon and can be stated as follows: if the magnetic field $H$ oscillates between two values, $H_{0}$ and $H_{1}$, then the representative point in the $(H, B)$-plane tends to a "limiting loop" which is independent of the initial magnetic state. Actually, our model exhibits such a property as it is shown in the following:

Theorem 7.1. Given an initial magnetic state $m_{i}=\left(H_{0}, \bar{H}_{0}, B_{0}\right)$, for every magnetic process $H$, starting from $m_{i}$ and oscillating between $H_{0}$ and $H_{1}$, we have that the magnetic induction $B$ tends to oscillate between two values $B_{0}^{\infty}$ and $B_{1}^{\infty}$ (independent of $B_{0}$ and $\bar{H}_{0}$ ) as the number of oscillations tends to infinity.

Proof. Consider an initial magnetic state $m_{i}=\left(H_{0}, \bar{H}_{0}, B_{0}\right)$ and a magnetic process $H^{(n)}$, of duration $d$, oscillating $n$ times between the values $H_{0}$ and $H_{1}=H_{0}+h$. Without loss of generality, we assume that $\bar{H}_{0}=H_{0}$. Because of the rate-independence of our model, we suppose $H^{(n)}$ to increase and decrease linearly in time, as shown in Fig. 3.

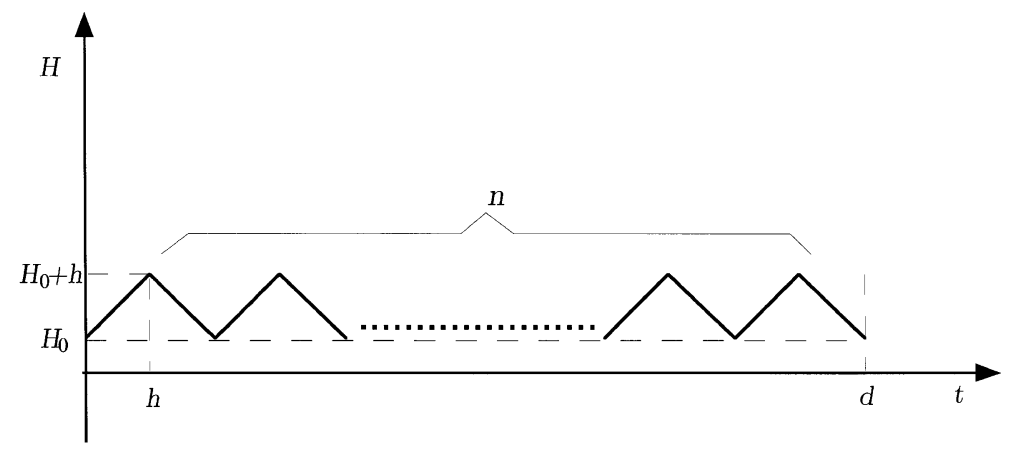

Fig. 3. The process $H^{(n)}$. 
Therefore, given the duration $d$, for any fixed $n \in \mathbb{N}$, we have that the oscillation amplitude $h$, the minimum points $H_{2 k}^{(n)}, k=0, \ldots, n$, and the maximum points $H_{2 k-1}^{(n)}, k=1, \ldots, n$, are given by

$$
h=\frac{d}{2 n}, \quad H_{2 k}^{(n)}=H_{0}, \quad H_{2 k-1}^{(n)}=H_{0}+h .
$$

Now, it is apparent that previous assumption $\bar{H}_{0}=H_{0}$ is quite general. In fact, we might consider as initial the state $m_{2}=\left(H_{2}^{(n)}, H_{2}^{(n)}, B_{2}\right)$ reached after the first oscillation $(k=2)$. Repeated applications of (7.3) provide the extremum values of $B$ after the $k$ th oscillation. Distinguishing between odd and even values of $k$, these extrema are given by

$$
\begin{aligned}
& B_{2 n-1}=b\left(H_{0}+h\right)+\left(B_{0}-b H_{0}\right) \mathrm{e}^{-(2 n-1) A(h)}-I(h) \frac{1+\mathrm{e}^{-(2 n-1) A(h)}}{1+\mathrm{e}^{-A(h)}}, \\
& B_{2 n}=B_{0} \mathrm{e}^{-2 n A(h)}+b H_{0}\left(1-\mathrm{e}^{-2 n A(h)}\right)+I(h) \frac{1-\mathrm{e}^{-2 n A(h)}}{1+\mathrm{e}^{-A(h)}} .
\end{aligned}
$$

Now, let us consider the limit of (7.5) as

$$
n \rightarrow \infty, \quad h=\text { const. }, \quad d=2 n h \rightarrow \infty
$$

Straightforward calculations yield

$$
\begin{aligned}
& B_{2 n} \rightarrow B_{0}^{\infty}=b H_{0}+I(h) \frac{1}{1+\mathrm{e}^{-A(h)}}, \\
& B_{2 n-1} \rightarrow B_{1}^{\infty}=b\left(H_{0}+h\right)-I(h) \frac{1}{1+\mathrm{e}^{-A(h)}} .
\end{aligned}
$$

Since both values are independent of the initial value $B_{0}$, the process described by (7.4) and (7.6) yields a limiting loop between the points $\left(H_{0}, B_{0}^{\infty}\right)$ and $\left(H_{0}+h, B_{1}^{\infty}\right)$.

Remark 7.2. The limiting loop reveals a lack of memory, since it is independent of the initial state. Moreover, this is obtained by means of a process with infinite duration and unbounded total variation. In fact, under conditions (7.6) we have

$$
\lim _{n \rightarrow \infty} \operatorname{Var}_{[0,2 h n]}\left(H^{(n)}-H_{0}\right)=\lim _{n \rightarrow \infty} 2 h n=+\infty
$$

Eqs. (7.7) can also be adapted to indicate the extrema of each primitive loop between $H_{0}$ and $H_{1}$, namely, the simplest closed loop having these two inversion points, only [3]. In fact, for every couple of values $H_{0}$ and $H_{1}$, the primitive hysteresis loop is uniquely provided by the inversion points $\left(H_{0}, B_{0}^{\infty}\right)$ and $\left(H_{1}, B_{1}^{\infty}\right)$, where $B_{0}^{\infty}$ and $B_{1}^{\infty}$ are given by (7.7) with $H_{1}=H_{0}+h$. Moreover, it is easy to check that such circles are counterclockwise oriented. In fact the slope of the loading curve reaching the inversion point is greater than the slope of the unloading curve starting from it (Fig. 4). In particular, 


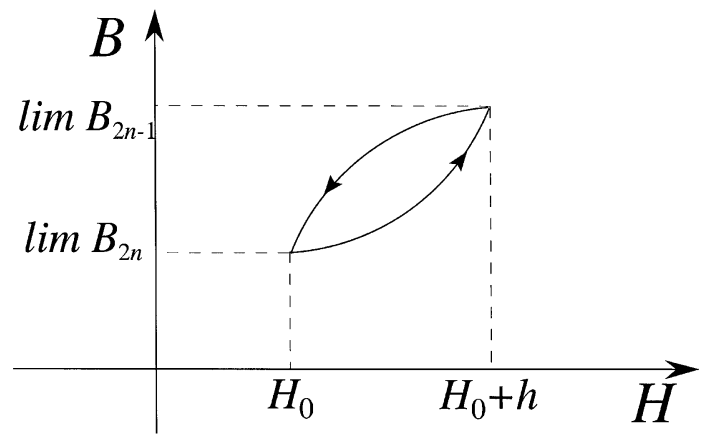

Fig. 4. The extrema of a limiting loop as a primitive loop.

$$
\left.\frac{\partial B}{\partial H}\right|_{H=\left(H_{0}+h\right)^{-}}-\left.\frac{\partial B}{\partial H}\right|_{H=\left(H_{0}+h\right)^{+}}=\left.\frac{\partial B}{\partial H}\right|_{H=H_{0}^{-}}-\left.\frac{\partial B}{\partial H}\right|_{H=H_{0}^{+}}=\alpha \sigma(h) I(h) \frac{1}{1+\mathrm{e}^{-A(h)}}>0,
$$

where the superscripts ' + ' and ' - ' denote the limiting values, respectively, for times grater and less than the inversion time.

Remark 7.3. Whatever $H_{0}$ may be, when $h \rightarrow 0$ the limiting loop shrinks to a single point, $\left(H_{0}, b H_{0}\right)$, which lies on the skeleton curve $B=f(H)$. This is the same property proved in [3]. However, unlike that and any other Duhem-type model, if we take the limit of (7.5) as $n \rightarrow \infty$ in such a way that $d$ remains constant, namely,

$$
h=\frac{d}{2 n} \text { and } \quad \underset{[0, d]}{\operatorname{Var}}\left(H^{(n)}-H_{0}\right)=d
$$

then we have

$$
B_{2 n} \rightarrow B_{0}, \quad B_{2 n-1} \rightarrow B_{0},
$$

so that the limiting loop contracts to the initial point. This is a distinguishing character of our model and we shall link it with vibro-stability in Section 7.3.

\subsection{A universal demagnetisation process}

For materials described by (7.1), we shall prove that a universal demagnetisation process does exist. In other words we can find a limiting process such that the material is asymptotically driven to the virgin state $m_{\mathrm{v}}=(0,0,0)$ whatever initial magnetic state $m_{0}=\left(H_{0}, \bar{H}_{0}, B_{0}\right)$ may be taken.

Consider an oscillating process $P_{u}$ such that the inversion points of $H$ are given by

$$
H_{k}=(-1)^{k} H_{0} d_{k},
$$


where $d_{k}>0$ are elements of a decreasing sequence such that

$$
d_{0}=1, \quad \lim _{k \rightarrow \infty} d_{k}=0 .
$$

As before, we restrict our attention to the region $\mathscr{M}^{*}$. In order to study the evolution equation (7.1) under the oscillating process $P_{u}$, the requirement $\left(H_{0}, B_{0}\right) \in \mathscr{M}^{*}$ is not restrictive. In fact, because of Eq. (7.9), if $H_{0} \notin\left[H^{*},-H^{*}\right]$, then there exists some $k^{*}$ such that $H_{k^{*}} \in\left[H^{*},-H^{*}\right]$, and we can consider as initial the value $H_{k^{*}}$ related to the first inversion point inside $\left[H^{*},-H^{*}\right]$.

According to (7.8), the distance between two consecutive inversion points of $P_{u}$, namely, $\Delta H_{k}=\left|H_{k}-H_{k-1}\right|$, is an alternating, decreasing sequence such that

$$
\Delta H_{k}=\left|(-1)^{k} H_{0}\left(d_{k}+d_{k-1}\right)\right|=\left|H_{0}\right|\left(d_{k}+d_{k-1}\right),
$$

and repeated applications of (7.3) provide the value of the magnetic induction $B_{n}$, corresponding to the $n$th inversion point $H_{n}$, is given by

$$
\begin{aligned}
B_{n}= & b H_{n}+\left(B_{0}-b H_{0}\right) \exp \left[-\sum_{k=1}^{n} A\left(\Delta H_{k}\right)\right]+\sum_{k=1}^{n}(-1)^{k} I\left(\Delta H_{k}\right) \exp \left[-\sum_{r=k}^{n} A\left(\Delta H_{r}\right)\right] \\
= & b H_{n}+\exp \left[-\sum_{k=1}^{n} A\left(\Delta H_{k}\right)\right]\left\{B_{0}-b H_{0}-I\left(\Delta H_{1}\right)\right. \\
& \left.+\sum_{k=2}^{n}(-1)^{k} I\left(\Delta H_{k}\right) \exp \left[\sum_{r=1}^{k-1} A\left(\Delta H_{r}\right)\right]\right\} .
\end{aligned}
$$

Introducing $\varphi_{n}$ and $S_{n}$ as

$$
\varphi_{0}=1, \quad \varphi_{n}=\exp \left[\sum_{r=1}^{n} A\left(\Delta H_{r}\right)\right], \quad S_{n}=\sum_{k=1}^{n}(-1)^{k} I\left(\Delta H_{k}\right) \varphi_{k-1},
$$

Eq. (7.11) can be rewritten as

$$
B_{n}=b H_{n}+\frac{1}{\varphi_{n}}\left(B_{0}-b H_{0}+S_{n}\right) .
$$

In order to give sufficient conditions on $P_{u}$ ensuring $B_{n} \rightarrow 0$ as $n \rightarrow \infty$, we prove the following lemmas.

Lemma 7.4. If the process $P_{u}$ is such that there exists a function $\rho: \mathbb{R}^{+} \rightarrow \mathbb{R}^{+}$satisfying

$$
\begin{aligned}
& \text { (h1) } \rho(s) \underset{s \rightarrow 0^{+}}{\longrightarrow} 0, \\
& \text { (h2) } \frac{s}{\rho(s)} \underset{s \rightarrow 0^{+}}{\searrow} 0,
\end{aligned}
$$


(h3) $\varphi_{k-1} \rho\left(\Delta H_{k}\right) \nearrow \infty$,

then, for some positive constant $C$, the following inequality holds:

$$
\left|S_{n}\right| \leqslant C \rho\left(\Delta H_{n}\right) \varphi_{n-1}
$$

Proof. Since the function $(1 / x) \int_{0}^{x} \mathrm{e}^{A(\eta)} \mathrm{d} \eta$ is bounded in a right neighbourhood of $x=0$, from (h2) we obtain

$$
\frac{1}{\rho(x)} \int_{0}^{x} \mathrm{e}^{A(\eta)} \mathrm{d} \eta=\frac{x}{\rho(x)} \cdot \frac{1}{x} \int_{0}^{x} \mathrm{e}^{A(\eta)} \mathrm{d} \eta \underset{s \rightarrow 0^{+}}{\searrow} 0
$$

so that the sequence

$$
G_{n}=(b-u) \sum_{k=1}^{n}(-1)^{k} \frac{1}{\rho\left(\Delta H_{k}\right)} \int_{0}^{\Delta H_{k}} \mathrm{e}^{A(\eta)} \mathrm{d} \eta
$$

converges as $n \rightarrow \infty$. Taking advantage of (h3) and using the identity

$$
\begin{aligned}
S_{n} & =(b-u)\left(\sum_{k=1}^{n}(-1)^{k} \int_{0}^{\Delta H_{k}} \mathrm{e}^{A(\eta)} \mathrm{d} \eta \varphi_{k-1}\right) \\
& =G_{n} \rho\left(\Delta H_{n}\right) \varphi_{n-1}+\sum_{k=1}^{n-1}\left(\rho\left(\Delta H_{k}\right) \varphi_{k-1}-\rho\left(\Delta H_{k+1}\right) \varphi_{k}\right) G_{k}
\end{aligned}
$$

it easily follows the estimate

$$
\begin{aligned}
\left|S_{n}\right| & \leqslant\left|G_{1}\right|\left(\rho\left(\Delta H_{n}\right) \varphi_{n-1}+\sum_{k=1}^{n-1}\left(\rho\left(\Delta H_{k+1}\right) \varphi_{k}-\rho\left(\Delta H_{k}\right) \varphi_{k-1}\right)\right) \\
& =\left|G_{1}\right|\left(2-\frac{\rho\left(\Delta H_{1}\right)}{\rho\left(\Delta H_{n}\right) \varphi_{n-1}}\right) \rho\left(\Delta H_{n}\right) \varphi_{n-1} \leqslant C \rho\left(\Delta H_{n}\right) \varphi_{n-1},
\end{aligned}
$$

That proves (7.13).

Lemma 7.5. There exists at least a process $P_{u}$ satisfying (7.8) and (7.9) and hypotheses (h1)-(h3) of Lemma 7.4. In particular, all requirements are fulfilled by choosing the sequence $\left\{d_{n}\right\}_{n \in \mathbb{N}}$ in (7.9) so that

$$
\Delta H_{n}=2\left|H_{0}\right| n^{-(p / 2)}, \quad p \in(0,1)
$$


Proof. Taking account of (5.9) it is easy to check that (7.14) implies

$$
\lim _{n \rightarrow \infty} \sum_{k=1}^{n} A\left(\Delta H_{k}\right)=+\infty
$$

and, for sufficiently large $n$,

$$
\Delta H_{n+1} \mathrm{e}^{(1 / p) A\left(\Delta H_{n}\right)}>\Delta H_{n} .
$$

The last inequality implies

$$
\left(\Delta H_{n+1}\right)^{p} \exp \left[\sum_{k=1}^{n} A\left(\Delta H_{k}\right)\right]>\left(\Delta H_{n}\right)^{p} \exp \left[\sum_{k=1}^{n-1} A\left(\Delta H_{k}\right)\right] .
$$

Therefore, the choice $\rho(x)=x^{p}$ trivially satisfies conditions (h1) and (h2), whereas (h3) is satisfied by virtue of (7.15) and (7.16). As a consequence (7.13) holds.

Theorem 7.6. For every oscillating process $P_{u}$ satisfying (7.8), (7.9) and (7.14), and whatever the initial state $m_{0}=\left(H_{0}, \bar{H}_{0}, B_{0}\right)$ may be, the related magnetic induction $B_{n}$ vanishes asymptotically, namely,

$$
\lim _{n \rightarrow \infty} B_{n}=0 .
$$

Thus, $P_{u}$ is called universal demagnetisation process since it leads the material to the virgin state $m_{\mathrm{v}}$ starting from any state $m_{0}$.

Proof. Note that from (7.8)-(7.10) it follows

$$
\lim _{n \rightarrow \infty} H_{n}=\lim _{n \rightarrow \infty} \Delta H_{n}=0 .
$$

and, moreover,

$$
\lim _{n \rightarrow \infty} \bar{H}_{n}=0 .
$$

On the other hand, (h1) and (h3) imply

$$
\lim _{n \rightarrow \infty} \varphi_{n}=+\infty
$$

and

$$
\frac{\left|S_{n}\right|}{\varphi_{n+1}} \leqslant \frac{C}{\mathrm{e}^{A\left(\Delta H_{n+1}\right)}} \rho\left(\Delta H_{n}\right) .
$$


Thus, it is easy to check that (7.17), (7.18) and (7.19) yield the asymptotical vanishing of $B$. Finally, the process here constructed is independent of the initial magnetic induction $B_{0}$, in fact it depends on the initial magnetic field $H_{0}$, only.

By virtue of the choice (7.14), it is worth noting that process $H_{u}=\hat{H}\left(H_{0}, P_{u}\right)$ has an unbounded total variation, namely,

$$
\lim _{T \rightarrow+\infty} \underset{[0, T]}{\operatorname{Var}}\left(H_{u}\right)=\sum_{k=0}^{\infty}\left|\Delta H_{k}\right|=\sum_{n=0}^{\infty} 2\left|H_{0}\right| n^{-(p / 2)}=+\infty \quad \forall p \in(0,1) .
$$

This fact and Remark 7.2 suggest that permanent memory (i.e., memory of the initial magnetisation $B_{0}$ ) fades as the total variation of $H$ goes to infinity. Similar results hold for the integral-type model proposed by Bouc in [1].

\subsection{Stability of stationary magnetic states}

Some authors claim that one of the main properties of a physically admissible model of hysteresis is the so-called vibro-stability, that means continuity of the hysteretic functional [12,18]. Unfortunately, we are not able to prove that our model satisfy this property. However, we prove here that a restricted statement is fulfilled, namely, the stability of all stationary magnetic states. This property prevents the model to exhibit a spontaneous magnetisation under small perturbations of bounded total variation.

Definition 7.7. A magnetic state $m_{s}=\left(H_{s}, \bar{H}_{s}, B_{s}\right)$ is called stationary if it remains unchanged under the action of the constant process, i.e., $H(t)=H_{s}, t \in(0, d), d>0$.

It is easy to check that $\bar{H}_{s}=H_{s}$ for every stationary state $m_{s}$. The main result of this section is the following:

Theorem 7.8. Given any stationary magnetic state $m_{s}=\left(H_{0}, H_{0}, B_{0}\right)$, the path $m(t)=(H(t)$, $\bar{H}(t), B(t))$, starting from $m_{s}$ and corresponding to the process $H \in L B V$ of finite duration $d$, satisfies the estimate

$$
\sup _{t \in(0, d)}\left|B(t)-B_{0}\right| \leqslant C_{\mathrm{V}} \sup _{t \in(0, d)}\left|H(t)-H_{0}\right|
$$

where $C_{\mathrm{V}}$ is a constant depending on the total variation of $H$ on $(0, d)$. Moreover, $C_{\mathrm{V}}$ is bounded if and only if $\operatorname{Var}_{[0, d]}\left(H-H_{0}\right)$ is bounded.

In order to prove Theorem 7.8, as before we restrict our attention to any initial stationary state $m_{s}=\left(H_{0}, H_{0}, B_{0}\right)$ such that $\left(B_{0}, H_{0}\right) \in \mathscr{M}^{*}$, and any process $H \in L B V$ whose values lie inside $\left[-H^{*}, H^{*}\right.$. Since $H$ has at most a numerable set of inversion points, we first consider the class of piecewise monotone processes $P_{m}$, with finite duration $d$ and whose sign changes at a finite number of time instants, namely, 


$$
0=t_{0}<t_{1}<\cdots<t_{n}=d .
$$

Accordingly, we denote

$$
H_{k}=H\left(t_{k}\right), \quad B_{k}=B\left(t_{k}\right), \quad \Delta H_{k}=\left|H_{k}-H_{k-1}\right| .
$$

In this framework, Theorem 7.8 can be restated as follows:

Theorem 7.8'. Starting from any stationary magnetic state $m_{s}=\left(H_{0}, H_{0}, B_{0}\right)$, every piecewise monotone process $H$, described by (7.21) and (7.22) and such that $\operatorname{Var}_{[0, d]}\left(H-H_{0}\right)<\infty$, leads to the following estimate:

$$
\left|B_{n}-B_{0}\right| \leqslant C_{\mathrm{V}} \sup _{t \in(0, d)}\left|H(t)-H_{0}\right|
$$

for some positive constant $C_{\mathrm{V}}$ depending on $\operatorname{Var}_{[0, d]}\left(H-H_{0}\right)$.

Before proving Theorem $7.8^{\prime}$ we give some preliminary results. Without loss of generality, we can choose the process $H$ such that the inversion points in (7.22) satisfy

$$
H_{2 k+1}>H_{2 k} \text { for } k=0,1, \ldots, r_{1}, \quad \text { and } H_{2 k-1}>H_{2 k} \text { for } k=1, \ldots, r_{2},
$$

where $r_{1}=r_{2}=(n-1) / 2$ if $n$ is odd and $r_{1}=n / 2-1, r_{2}=n / 2$ if $n$ is even. By virtue of (7.3) the magnetic induction at the inversion instants $t_{2 k}$ and $t_{2 k+1}$ are, respectively, given by

$$
\begin{aligned}
& B_{2 k}=b H_{2 k}+\mathrm{e}^{-\Delta H_{2 k}}\left[B_{2 k-1}-b H_{2 k-1}+I\left(\Delta H_{2 k}\right)\right], \\
& B_{2 k+1}=b H_{2 k+1}+\mathrm{e}^{-\Delta H_{2 k+1}}\left[B_{2 k}-b H_{2 k}-I\left(\Delta H_{2 k+1}\right)\right] .
\end{aligned}
$$

By recurrence, (7.24) imply

$$
\begin{aligned}
B_{n}-B_{0}= & b\left(H_{n}-H_{0}\right)+\left(B_{0}-b H_{0}\right)\left[\exp \left[-\sum_{k=1}^{n} A\left(\Delta H_{k}\right)\right]-1\right] \\
& +\sum_{k=1}^{n}\left[(-1)^{k} I\left(\Delta H_{k}\right) \exp \left[-\sum_{r=k}^{n} A\left(\Delta H_{r}\right)\right]\right] .
\end{aligned}
$$

The second addendum can be estimated using the inequality

$$
1-\exp \left[-\sum_{k=1}^{n} A\left(\Delta H_{k}\right)\right] \leqslant \sum_{k=1}^{n} A\left(\Delta H_{k}\right) .
$$

In order to estimate the last addendum in (7.25), namely,

$$
\Gamma_{n}=\sum_{k=1}^{n}\left[(-1)^{k} I\left(\Delta H_{k}\right) \exp \left[-\sum_{r=k}^{n} A\left(\Delta H_{h}\right)\right]\right],
$$

we prove the following: 
Lemma 7.9. For any piecewise monotone process $H$, the following estimate holds:

$$
\left|\Gamma_{n}\right| \leqslant \sup \left|H-H_{0}\right|+\underset{[0, d]}{\operatorname{Var}}\left(H-H_{0}\right) \sum_{k=1}^{n} A\left(\Delta H_{k}\right) .
$$

Proof. Note that each integral $I\left(\Delta H_{k}\right)$ can be rewritten as

$$
I\left(\Delta H_{k}\right)=(-1)^{k+1}(b-u) \int_{t_{k-1}}^{t_{k}} \mathrm{e}^{A\left(H(t)-H_{k-1}\right)} \dot{H} \mathrm{~d} t
$$

Then, (7.27) is equivalent to

$$
\Gamma_{n}=\int_{0}^{d} \exp \left[-\sum_{r=k(t)-1}^{n}\left[A\left(\Delta H_{r}\right)-A\left(H(t)-H_{k(t)-1}\right)\right]\right] \dot{H} \mathrm{~d} t
$$

where the integer $k(t)$ depends on the variable of integration $t \in[0, d]$ in the sense that

$$
k(t)=k \quad \text { if and only if } t_{k-1}<t \leqslant t_{k} .
$$

By virtue of (7.29) and (7.26), $\Gamma_{n}$ can be estimated as

$$
\begin{aligned}
\left|\Gamma_{n}\right| & \leqslant\left|\int_{0}^{d} \dot{H} \mathrm{~d} t\right|+\int_{0}^{d}\left|1-\exp \left[-\sum_{r=k(t)-1}^{n}\left[A\left(\Delta H_{r}\right)-A\left(H(t)-H_{k(t)-1}\right)\right]\right]\right| \dot{H} \mid \mathrm{d} t \\
& \leqslant\left|\int_{0}^{d} \dot{H} \mathrm{~d} t\right|+\int_{0}^{d}\left|\sum_{r=k(t)-1}^{n}\left[A\left(\Delta H_{r}\right)-A\left(H(t)-H_{k(t)-1}\right)\right]\right||\dot{H}| \mathrm{d} t \\
& \leqslant \sup \left|H-H_{0}\right|+\left(H-H_{0}\right) \sum_{k=1}^{n} A\left(\Delta H_{k}\right)
\end{aligned}
$$

that immediately yields (7.28).

Proof of Theorem 7.8'. By virtue of (7.24), (7.26) and (7.28) we have

$$
\left|B_{n}-B_{0}\right| \leqslant(1+b) \sup \left|H-H_{0}\right|+\left(\left|B_{0}-b H_{0}\right|+\underset{[0, d]}{\operatorname{Var}}\left(H-H_{0}\right)\right) \sum_{k=1}^{n} A\left(\Delta H_{k}\right) .
$$

Moreover, by virtue of (5.9), it is easy to check the following estimate:

$$
\sum_{k=1}^{n} A\left(\Delta H_{k}\right) \leqslant \frac{1}{2} \alpha \sigma^{\prime}(0) \sum_{k=1}^{n}\left(\Delta H_{k}\right)^{2} \leqslant \alpha \sigma^{\prime}(0) \sup \left|H-H_{0}\right| \underset{[0, d]}{\operatorname{Var}}\left(H-H_{0}\right) .
$$


Finally, by virtue of (7.30) and (7.31), we obtain inequality (7.23) with $C_{\mathrm{V}}$ expressed by

$$
C_{\mathrm{V}}=1+b+\alpha \sigma^{\prime}(0)\left[\left|B_{0}-b H_{0}\right|+\underset{[0, d]}{\operatorname{Var}}\left(H-H_{0}\right)\right] \underset{[0, d]}{\operatorname{Var}\left(H-H_{0}\right)},
$$

which is bounded if and only if $\operatorname{Var}_{[0, d]}\left(H-H_{0}\right)$ is bounded too.

Theorem 7.8 means that all stationary states are stable with respect to the $C^{0}(0, d)$-norm. Nevertheless, $C_{\mathrm{V}}$ depends on the total variation of $H-H_{0}$, so that stability is related to processes yielding continuous magnetic histories with bounded total variation, namely,

$$
H \in C^{0}([0, d]) \cap B V(0, d) .
$$

It is worth noting that $H \in B V(0, d)$ is a requirement consistent with $H^{t} \in L B V$. In addition, it is not surprising. In fact, we previously observed that magnetic histories with unbounded total variation lead to the lack of memory effects for $B$, so that the material may exhibit limiting loops or a universal demagnetisation processes. In this connection, it would be very anomalous if stability, or even vibro-stability, did not depend in some way on the total variation of the process.

\section{Acknowledgements}

The authors wish to thank M. Fabrizio and A. Morro for helpful discussions. This work has been done under the auspices of G.N.F.M. of I.N.D.A.M., partially supported by the Italian MURST through the COFIN'98 Research Program “Modelli Matematici per la Scienza dei Materiali" and partially supported by University of Bologna Funds for selected research topics.

\section{References}

[1] R. Bouc, Acustica 24 (1971) 16.

[2] M. Brokate, Appl. Anal. 39 (1989) 229.

[3] B.D. Coleman, M.L. Hodgdon, Int. J. Eng. Sci. 24 (1986) 897.

[4] B.D. Coleman, M.L. Hodgdon, Arch. Rational Mech. Anal. 99 (1987) 375.

[5] A. Damlamian, A. Visintin, CR Acad. Sci. Paris (I) 297 (1983) 437.

[6] M. Fabrizio, C. Giorgi, Boll. UMI Suppl. Fisica Mat. (Suppl. 1) (1981) 33.

[7] C. Heck, Magnetic Materials and Their Applications, Crane, Russak, New York, 1974.

[8] M. Hilpert, in: Mathematical Models for Phase Change Problems (Obidos 1988), Birkhauser, Basel, Int. Ser. Numer. Math. 88 (1989) 377.

[9] M.L. Hodgdon, IEEE Trans. Magn. MAG-24 (1988) 218.

[10] M.L. Hodgdon, IEEE Trans. Magn. MAG-24 (1988) 3120.

[11] M.A. Krasnosel'skii, Soviet Math. Dokl. 11 (1970) 1510.

[12] M.A. Krasnosel'skii, A.V. Pokrovskii, Systems with Hysteresis, Springer, Berlin, 1989.

[13] P. Krejci, Math. Z. 193 (1986) 247.

[14] J.W. Macki, P. Nistri, P. Zecca, SIAM Rev. 35 (1993) 94.

[15] I.D. Mayergoyz, The Preisach Model for Hysteresis, Springer, Berlin, 1991.

[16] D.R. Owen, W.O. Williams, Quart. Appl. Math. 26 (1968) 321. 
[17] A.C. Pipkin, R.S. Rivlin, Z. Angew. Math. Phys. 16 (1965) 313.

[18] A. Visintin, Differential Models of Hysteresis, Springer, Berlin, 1994.

[19] A. Visintin, Nonlinear Anal. TMA 8 (1984) 977.

[20] A. Visintin, in: Proceedings of the Sixth International Symposium on Computing Methods in Applied Sciences and Engineering, vol. VI, Versailles, 1983, North-Holland, Amsterdam, 1984, p. 149.

[21] A. Visintin, SIAM J. Appl. Math. 17 (1986) 1113.

[22] A. Visintin, Atti Sem. Mat. Fis. Univ. Modena 32 (1983) 232. 